\title{
Neutrophilic inflammation during lung development disrupts elastin assembly and predisposes adult mice to COPD
}

\author{
John T. Benjamin, ${ }^{1}$ Erin J. Plosa, ${ }^{1}$ Jennifer M.S. Sucre, ${ }^{1,2}$ Riet van der Meer, ${ }^{1}$ Shivangi Dave, ${ }^{1}$ Sergey Gutor, ${ }^{3}$ David S. Nichols, ${ }^{3}$ \\ Peter M. Gulleman, ${ }^{1}$ Christopher S. Jetter, ${ }^{1}$ Wei Han, ${ }^{3}$ Matthew Xin, ${ }^{3}$ Peter C. Dinella, ${ }^{1}$ Ashley Catanzarite, ${ }^{1}$ Seunghyi Kook, ${ }^{1}$ \\ Kalsang Dolma, ${ }^{4}$ Charitharth V. Lal, ${ }^{4}$ Amit Gaggar, ${ }^{5,6,7}$ J. Edwin Blalock, ${ }^{5,6,7}$ Dawn C. Newcomb, ${ }^{3}$ Bradley W. Richmond, ${ }^{3,8}$ \\ Jonathan A. Kropski, ${ }^{2,3,8}$ Lisa R. Young, ${ }^{1,2,3,9}$ Susan H. Guttentag,' and Timothy S. Blackwell ${ }^{2,3,8}$
}

'Department of Pediatrics, Division of Neonatology, Vanderbilt University Medical Center, Nashville, Tennessee, USA. Department of Cell and Developmental Biology, Vanderbilt University Medical Center, Nashville, Tennessee, USA. ${ }^{3}$ Department of Medicine, Division of Allergy, Pulmonary, and Critical Care Medicine, Vanderbilt University Medical Center, Nashville, Tennessee, USA. ${ }^{4}$ Department of Pediatrics, University of Alabama at Birmingham, Birmingham, Alabama, USA. ${ }^{5}$ Department of Medicine, Division of Pulmonary, Allergy and Critical Care, University of Alabama at Birmingham, Birmingham, Alabama, USA. ${ }^{6}$ Program in Protease and Matrix Biology, University of Alabama at Birmingham, Birmingham, Alabama, USA. ${ }^{~ B i r m i n g h a m ~ V e t e r a n s ~ A f f a i r s ~ M e d i c a l ~ C e n t e r, ~ B i r m i n g h a m, ~ A l a b a m a, ~ U S A . ~}{ }^{8}$ Nashville Veterans Affairs Medical Center, Nashville, Tennessee, USA. ${ }^{9}$ Department of Pediatrics, Division of Pulmonary Medicine, Children's Hospital of Philadelphia and the Perelman School of Medicine at the University of Pennsylvania, Philadelphia, Pennsylvania, USA.

\begin{abstract}
Emerging evidence indicates that early life events can increase the risk for developing chronic obstructive pulmonary disease (COPD). Using an inducible transgenic mouse model for NF- $\mathrm{B}$ B activation in the airway epithelium, we found that a brief period of inflammation during the saccular stage (P3-P5) but not alveolar stage (P10-P12) of lung development disrupted elastic fiber assembly, resulting in permanent reduction in lung function and development of a COPD-like lung phenotype that progressed through $\mathbf{2 4}$ months of age. Neutrophil depletion prevented disruption of elastic fiber assembly and restored normal lung development. Mechanistic studies uncovered a role for neutrophil elastase (NE) in downregulating expression of critical elastic fiber assembly components, particularly fibulin-5 and elastin. Further, purified human NE and NE-containing exosomes from tracheal aspirates of premature infants with lung inflammation downregulated elastin and fibulin- 5 expression by saccular-stage mouse lung fibroblasts. Together, our studies define a critical developmental window for assembling the elastin scaffold in the distal lung, which is required to support lung structure and function throughout the lifespan. Although neutrophils play a well-recognized role in COPD development in adults, neutrophilic inflammation may also contribute to early-life predisposition to COPD.
\end{abstract}

\section{Introduction}

Although the traditional view has been that cigarette smoking causes chronic obstructive pulmonary disease (COPD) through accelerated age-related lung-function decline in susceptible smokers, over the last several years it has become apparent that up to $50 \%$ of COPD may be related to reduced peak lung function in young adulthood without accelerated decline (1). In this regard, early life events have been associated with reduced adult lung function, which peaks between 20 and 25 years of age $(2,3)$. Bronchopulmonary dysplasia (BPD) is a chronic respiratory disorder of premature infants born in the saccular stage of lung development that is characterized by dilated and fewer terminal airspaces $(4,5)$. Several recent studies have shown that patients with BPD have impaired lung function in adolescence and young adulthood compared with preterm infants without $\mathrm{BPD}$ or term infants $(4,6,7)$. In addition, radiographic abnormalities,

Conflict of interest: The authors have declared that no conflict of interest exists. Copyright: @ 2021, American Society for Clinical Investigation.

Submitted: April 23, 2020; Accepted: October 21, 2020; Published: January 4, 2021.

Reference information: / Clin Invest. 2021;131(1):e139481.

https://doi.org/10.1172/JCl139481. including emphysema and air trapping, have been reported on CT scans from adults with a history of BPD (8). This association between BPD and altered lung function trajectory points to the saccular stage as an important developmental window for early life events that permanently affect lung structure and function.

Data from human studies and animal experiments indicate that inflammation in the preterm lung can alter its development (9). Infants who are exposed to chorioamnionitis in utero are likely to be born preterm and many develop BPD (10). Similarly, infections in the early postnatal period increase BPD risk in premature infants (11). Previously, we reported that perinatal transgene activation in mice engineered to express an inducible form of IкB-kinase $\beta$ (IKK- $\beta$ ), the upstream activator of the master inflammatory transcription factor $\mathrm{NF}-\kappa \mathrm{B}$, in airway epithelial cells results in early postnatal death with hypoxemia, dilation of terminal saccules, and disorganized elastic fiber formation (12). Based on these findings, we hypothesized that transient NF- $\mathrm{BB}$-induced inflammation during the saccular stage of lung development could disrupt formation of the elastin scaffold of the distal lung, resulting in permanently impaired lung function. Therefore, we modified this model to induce sublethal inflammation and injury during either the saccular 
(P3-P5) or alveolar stage (P10-P12) of mouse lung development. Remarkably, epithelium-derived inflammation in the saccular but not alveolar stage resulted in an early BPD-like phenotype with disruption of the developing elastin meshwork in the distal lung, followed by emphysematous airspace dilation with reduced lung function that persisted through 24 months of age. These studies define a critical window in saccular-stage lung development for establishing the elastin scaffold required to support distal lung structure and function throughout the lifespan.

\section{Results}

$N F-\kappa B$ activation in the saccular stage results in permanent abnormalities in lung structure and function. In mice with doxycycline-inducible (Dox-inducible) expression of constitutively active human IKK- $\beta$ in airway epithelium (called IKTA mice) (13), we induced transient transgene expression in pups during lung development by treating lactating dams with Dox in drinking water from P3 to P5 (saccular) or P10 to P12 (alveolar). We then examined lungs of IKTA and littermate control mice over time. At 2 months of age, lungs of adult IKTA mice with Dox administration from P3 to P5 showed marked emphysematous changes throughout the lung parenchyma (Figure $1, A$ and B). In contrast, lungs of adult IKTA mice with Dox from P10 to P12 appeared similar to control lungs on histological evaluation. In addition, small airways from IKTA mice with P3-P5 Dox exhibited a significant reduction in the number of radial alveolar attachments (Figure 1, C and D), which are connective tissue-containing septa that insert radially into the adventitia of small airways and are responsible for tethering to adjacent alveolar tissue (14).

We next evaluated elastic fibers in the lungs of 2-month-old IKTA mice and found that elastic fibers were fragmented and reduced in number throughout the lung parenchyma of 2-monthold IKTA mice with Dox administration from P3 to P5, whereas 2 month-old IKTA mice with Dox from P10 to P12 demonstrated normal cord-like arrangement of elastic fibers around distal airspaces, similar to those seen in littermate controls (Figure 1E). Together, these data suggest that brief periods of NF- $\kappa \mathrm{B}-$ induced inflammation during saccular-stage lung development disrupted assembly of the elastic fiber scaffold, resulting in long-term pathological alterations in the distal lung parenchyma.

Given that elastic fibers have a very long half-life and their assembly is developmentally regulated $(15,16)$, we followed IKTA mice (Dox P3-P5) to 24 months of age to investigate the lifelong consequences of saccular-stage lung inflammation. At 24 months, elderly IKTA mice (Dox P3-P5) had a striking emphysematous phenotype that was visible at the time of lung harvest and on lung sections, along with persistent elastic fiber fragmentation and disorganization (Figure 2, A-C). Flow cytometry for immune cells and mRNA expression of inflammatory cytokines revealed no evidence of ongoing inflammation in IKTA mice (Dox P3-P5) at 3 or 24 months of age compared with controls (Supplemental Figure 1), thereby suggesting that progressive emphysematous changes in these mice did not result from persistent lung inflammation.

To evaluate the trajectory of structural changes in the lung parenchyma of IKTA mice with saccular-stage transgene activation, we performed morphometric evaluation of lungs from IKTA mice (Dox P3-P5) and littermate controls at 2, 6, and 24 months of age. As shown in Figure 2, D and E, progressive alveolar enlargement measured by mean linear intercept (MLI) and reduction in alveolar attachment counts were identified from 2 to 24 months of age in littermate control mice, demonstrating the effects of aging; however, IKTA mice (Dox P3-P5) had increased MLI and reduced alveolar attachments at each time point compared with controls. Collectively, these findings indicate that impaired elastic fiber organization during saccular lung development permanently altered lung structure and, with the effects of aging, resulted in progressive worsening of emphysema and alveolar attachment loss.

To investigate the functional consequences of altered lung structure in IKTA mice (Dox P3-P5), we measured lung mechanics by FlexiVent (SCIREQ). Compared with littermate controls and IKTA mice with transgene activation during the alveolar stage (Dox P10-P12), 2-month-old IKTA mice with Dox from P3 to P5 had reduced lung elastance, reduced respiratory system elastance, and increased lung compliance (Supplemental Figure 2). As mice aged from 2 to 24 months, elastance decreased and compliance increased in control mice, but IKTA mice (Dox P3-P5) had reduced lung tissue elastance and respiratory system elastance, along with increased compliance, at each time point compared with littermate controls (Figure 2, F-H). As an additional control group, we evaluated untreated IKTA mice (never exposed to Dox) at 24 months of age and found that lung histology was identical to littermate controls (Supplemental Figure 3A). Also, compared with both littermate controls and non-Dox-treated IKTA mice, 24-month-old IKTA mice (Dox P3-P5) had reduced lung tissue elastance, reduced respiratory system elastance, increased lung compliance, and increased inspiratory capacity (Supplemental Figure 3, B-E). Thus, alterations in lung function mirrored progression of emphysematous changes in lungs of IKTA mice subjected to saccular-stage lung inflammation.

Transgene activation in IKTA mice results in neutrophilic inflammation during the saccular and alveolar stages of lung development, but elastin fiber assembly is impaired only during the saccular stage. To investigate the short-term effects of transgene activation in IKTA mice during lung development, dams were placed on Dox in drinking water from P3 to P5 (saccular stage) or P10-P12 (alveolar stage) and lungs were examined at P5 or P12, respectively. As expected, transgene expression was detected in IKTA lungs at P5 and P12 (Figure 3A). IKTA lungs at both time points showed a neutrophil-predominant inflammatory cell influx (Figure 3, B and C). Consistent with the inflammatory cell influx at P5 and P12, expression of inflammatory cytokines was robustly induced in Dox-treated IKTA lungs at both P5 and P12 compared with controls (Figure 3, D and E).

At P5, abnormal lung development with dilated airspaces and aberrant organization of elastic fibers was apparent in IKTA mice (Dox P3-P5) (Figure 4, A-C). Impaired elastic fiber assembly correlated with reduced mRNA expression of elastin (Eln) and fibulin-5 (Fbln5), but not other critical components of elastic fiber assembly in P5 IKTA lungs (Figure 4D and Supplemental Figure $4 \mathrm{~A})$. In contrast, lung development was not altered at P12 in IKTA mice with Dox from P10 to P12 (Figure 4, E and F). Both elastic fiber organization and expression of $E \ln , F b \ln 5$, and other critical components of elastic fiber assembly were similar between P12 control and IKTA lungs (Figure 4, G and H, and Supplemental Figure 4B). Collectively, these data showed that neutrophilic inflammation selectively downregulated expression of Fbln 5 and Eln and altered elastic fiber organization in saccular-stage lungs. 
A

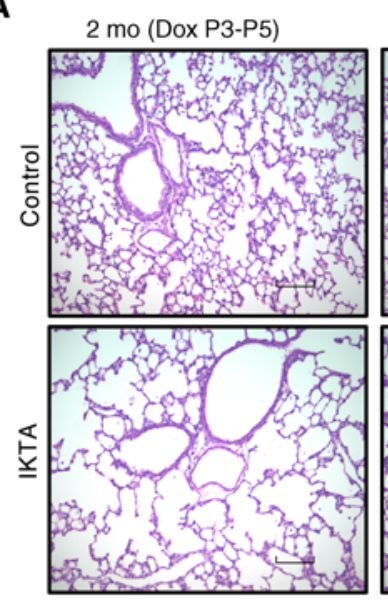

C

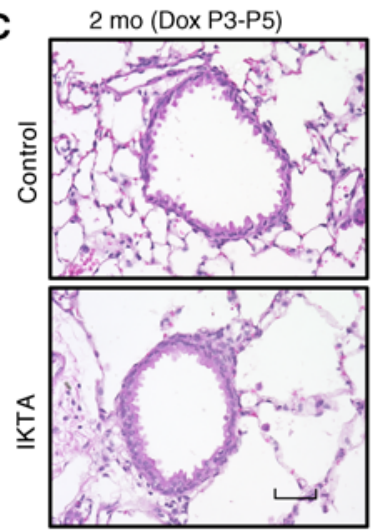

2 mo (Dox P10-P12)
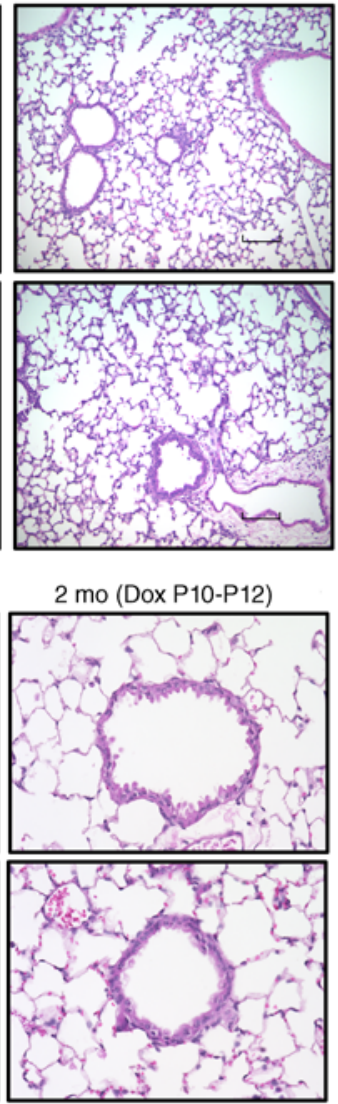

B
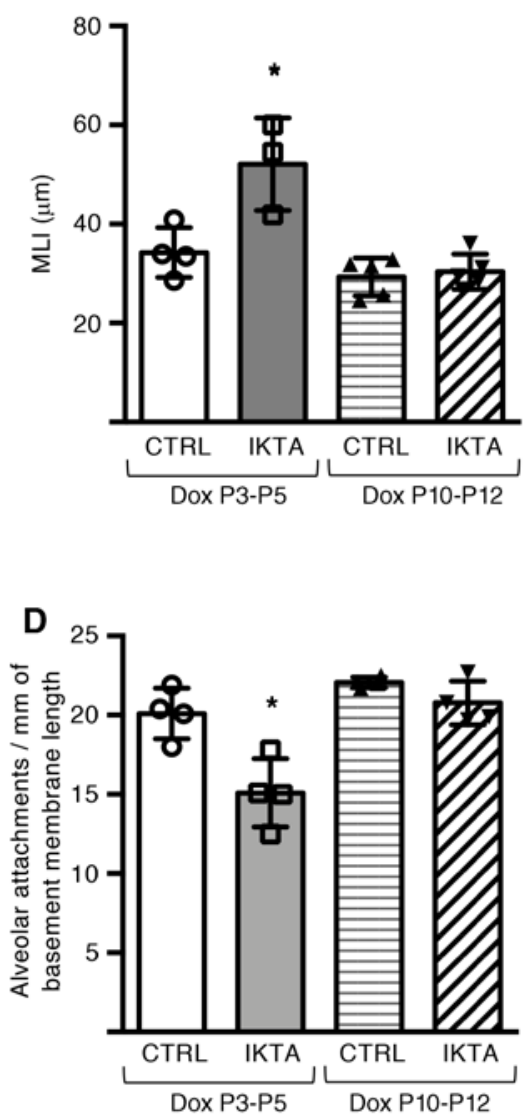

E
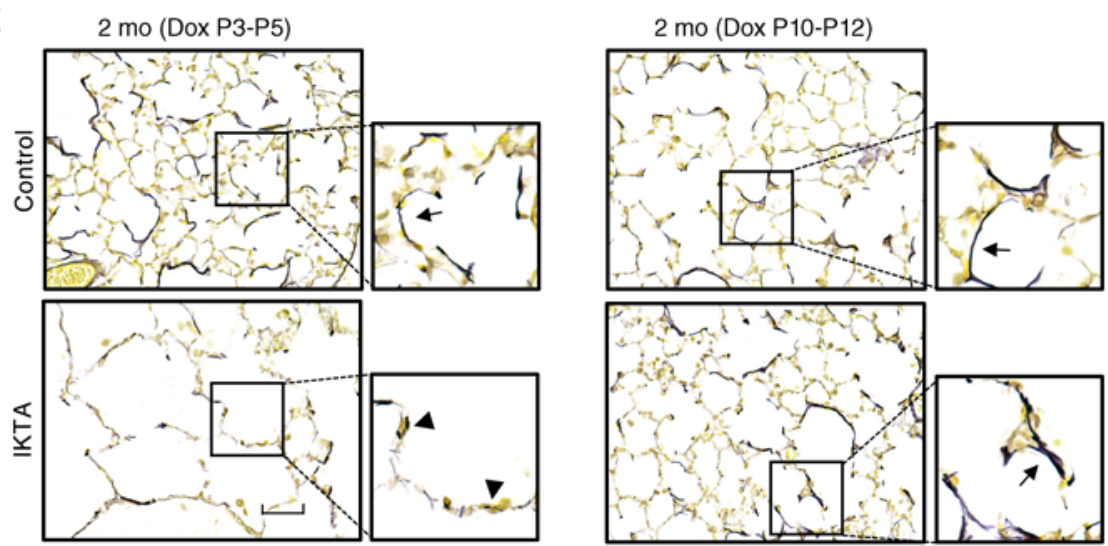

Figure 1. Epithelial NF-кB activation in saccular-stage lungs results in emphysema, loss of alveolar attachments, and disruption of elastic fiber organization. Dams were treated with Dox from P3 to P5 (saccular stage) or P10 to P12 (alveolar stage), after which lungs from IKTA and littermate control mice were harvested at 2 months of age. (A) Representative H\&E staining and (B) morphometric evaluation of emphysema as measured by mean linear intercept (MLI). Data are expressed as mean SEM, $n=3-4$ lungs per group, 5-6 imaged lung fields per lung. ${ }^{*} P<0.05$ by 1 -way ANOVA and post hoc Tukey test. (C) Representative H\&Estained sections showing small airways (arrows denote alveolar attachments) and (D) alveolar attachment counts in control and IKTA lungs. Data are expressed as mean \pm SEM, $n=3$ lungs per group, $P<0.05$ by 1-way ANOVA and post hoc Tukey test. (E) Representative photomicrographs of Hart's elastin-stained lung sections. Arrows denote elastic fibers around airspaces. Arrowhead denotes fragmented elastic fibers. Scale bar: $100 \mu \mathrm{m}$ in $\mathbf{A}, 50 \mu \mathrm{m}$ in $\mathbf{C}$ and $\mathbf{E}$.

Given that parenchymal growth in the saccular lung is closely related to vascular growth around distal airspaces (17), we examined whether vasculogenesis was altered in IKTA mice (Dox P3P5). Immunostaining for vWF, an endothelial cell marker, in P5 lungs revealed no differences in vessel density between Dox-treated control and IKTA lungs. Expression of the angiogenic growth factor Vegfa and its primary receptor Flk1 (Vegfr2) were also similar between Dox-treated control and IKTA lungs at P5 (Supplemental Figure 5, A and B). In addition, immunostaining for $\alpha$-smooth muscle actin ( $\alpha$-SMA) showed well-organized smooth muscle cells around large vessels in control and IKTA lungs, along with intact elastic lamina (Supplemental Figure 5, C and D). These findings 
A

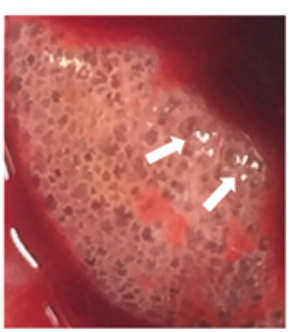

D

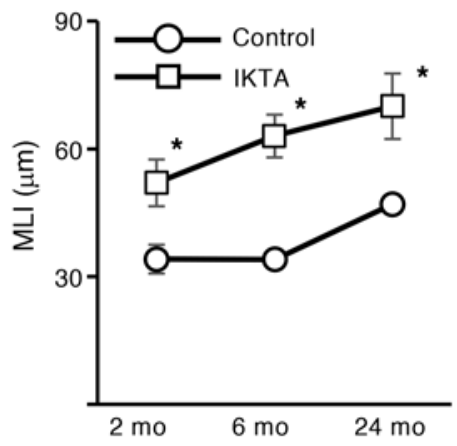

$\mathbf{F}$

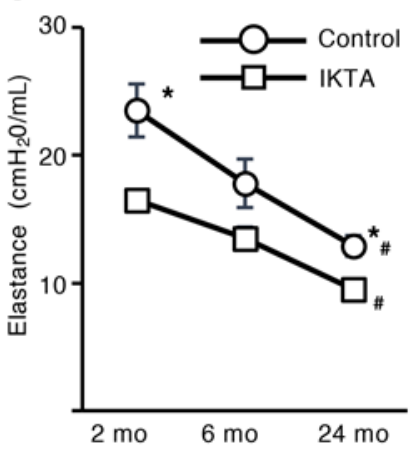

B
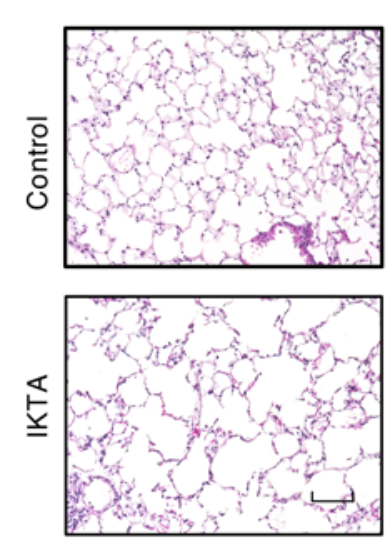

E

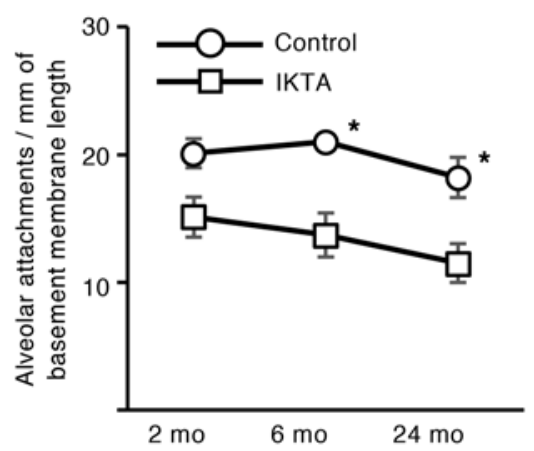

G

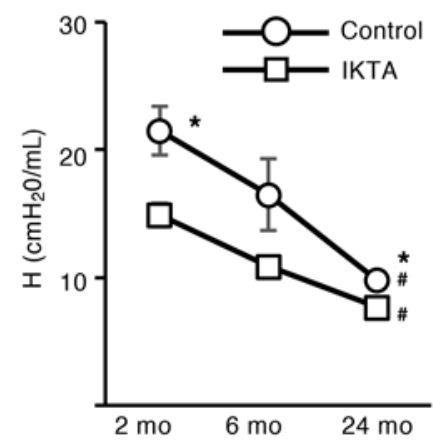

C
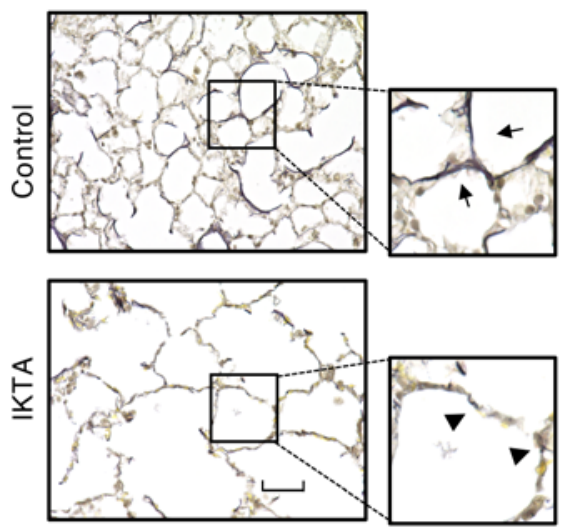

Figure 2. Epithelial NF- $\kappa B$ activation in saccular-stage lungs results in lifelong abnormalities in lung structure and function. Dams were treated with Dox from P3 to P5 (saccular stage), after which lungs from IKTA and littermate control mice were harvested at 2, 6, or 24 months. (A) Representative image showing gross appearance of a 24-month IKTA lung. Arrows denote areas of emphysema. (B and C) Representative images showing H\&E-stained (B) and Hart's elastin-stained (C) lung sections from 24-month control and IKTA lungs. Arrows denote intact elastic fibers. Arrowhead denotes fragments of elastic fibers. (D and E) Quantification of mean linear intercept (MLI) (D) and alveolar attachments (E) in control and IKTA lungs at 2, 6, and 24 months. Data are expressed as mean \pm SEM, $n=3-4$ lungs per group, 6 imaged lung fields per lung ${ }^{*} P<0.05$ by 2 -tailed Student's $t$ test comparing IKTA to littermate controls at each age. $(\mathbf{F}-\mathbf{H})$ Lung function, including total respiratory system elastance $(\mathbf{F})$, tissue elastance $(\mathbf{C})$, and dynamic compliance $(\mathbf{H})$, was measured at 2,6 , and 24 months in control and IKTA mice. Data are expressed as mean \pm SEM, $n=4-6$ mice per group, ${ }^{*} P<0.05$ by 2-tailed Student's $t$ test comparing IKTA to littermate controls at each age, ${ }^{\# P}<0.05$ by 1-way ANOVA and post hoc linear trend test for each group (control and IKTA). Scale bar: $100 \mu \mathrm{m}$ in B, $50 \mu \mathrm{m}$ in $\mathbf{C}$.

indicate that inflammation-induced changes in IKTA lungs primarily affected structural support for developing saccules without impairment of vasculogenesis.

Since saccular-stage transgene activation resulted in a profound developmental phenotype in IKTA mice, we wondered whether lung inflammation during this time frame altered proliferation or survival of parenchymal cell populations in the developing lung. Evaluation of proliferating cell nuclear antigen-positive (PCNA-positive) cells in P5 (Dox P3-P5) control and IKTA lung sections revealed no differences in proliferation of type 2 alveolar epithelial cells or other parenchymal cells in Dox-treated IKTA compared with control lungs (Supplemental Figure 6, A-C). We also performed TUNEL staining of P5 control and IKTA lung sections and found similar numbers of TUNEL ${ }^{+}$cells in both groups (Supplemental Figure 6D). Thus, altered lung development in IKTA mice did not appear to result from major shifts in proliferation or survival of nonimmune cell populations.

Neutrophil depletion rescues lung development in mice with saccular-stage inflammation. Since neutrophils predominated in the 
A

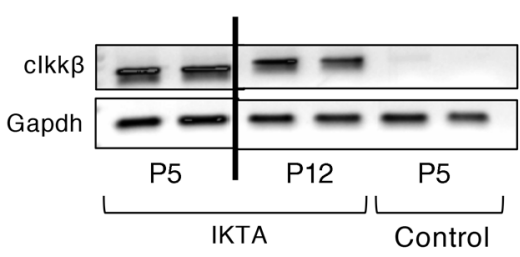

D

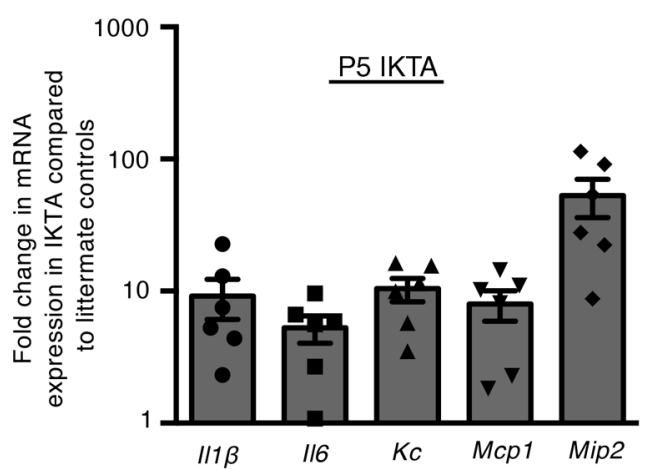

B

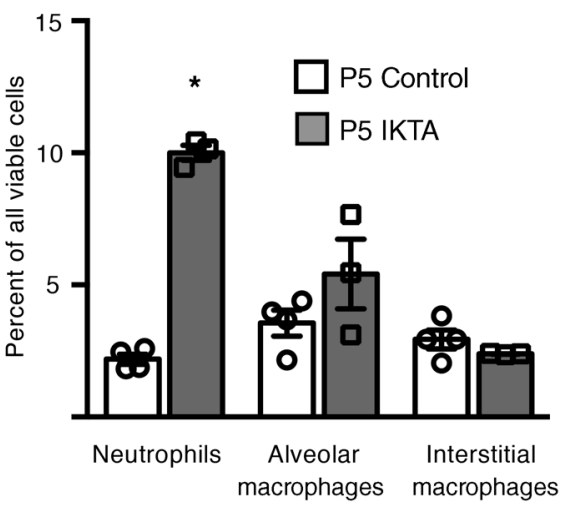

C

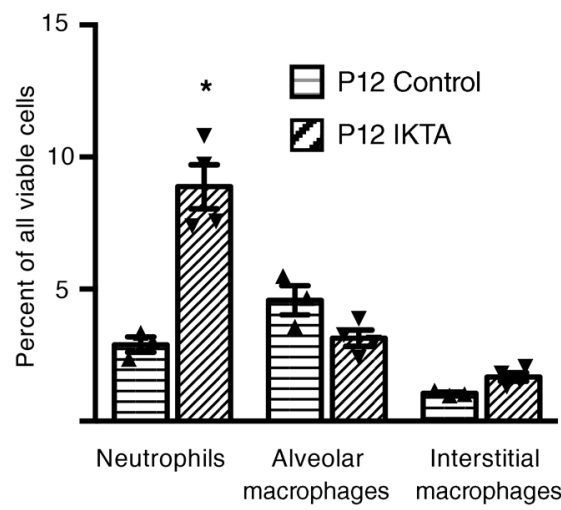

E

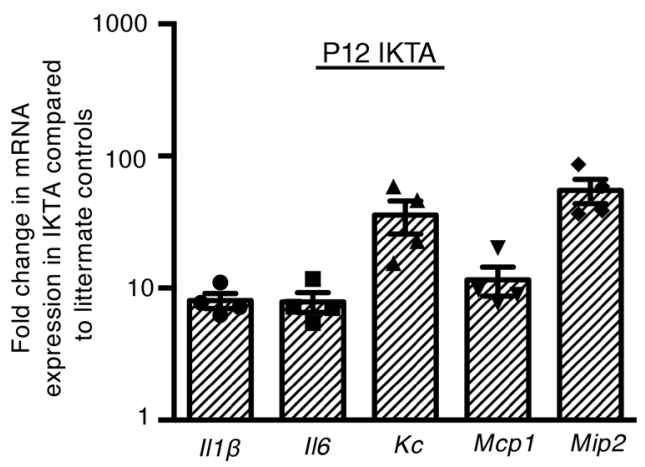

Figure 3. Transgene activation results in neutrophilic inflammation during both the saccular and alveolar stages of lung development. Dams were treated with Dox from P3 to P5 (saccular stage) or P10 to P12 (alveolar stage), after which lungs from IKTA and littermate control mice were harvested at P5 or P12. (A) Expression of constitutively active IKK- $\beta$ (clkk- $\beta$ ) transgene and Gapdh was evaluated by PCR followed by agarose gel electrophoresis. (B and C) Quantification of immune cells in P5 (B) and P12 (C) control and IKTA lungs by flow cytometry. Data are expressed as mean \pm SEM, $n=3-4$ per group. ${ }^{*} P<0.05$ by 2-tailed Student's $t$ test. (D and E) mRNA expression of select inflammatory cytokines in P5 (D) and P12 (E) control and IKTA lungs. Data are expressed as mean \pm SEM, $n=4-6$ per group.

inflammatory phenotype of saccular-stage IKTA mice, we administered anti-Ly6Gantibodies (orcontrolIgG) toIKTAandlittermatecontrol mice by i.p. injection on $\mathrm{P} 2$ in order to block the neutrophil influx induced by Dox treatment from P3 to P5. Flow cytometry analysis (Figure 5, A and B) and immunostaining for Gr-1 (Supplemental Figure 7) confirmed reduced neutrophil recruitment in Dox-treated IKTA lungs at P5 after administration of anti-Ly6G antibodies.

Neutrophil depletion normalized lung development in IKTA mice (Dox P3-P5), whereas control IgG had no effect on the BPDlike phenotype (Figure 5, C-E). To test whether neutrophil depletion corrects long-term lung development in IKTA mice (Dox P3-P5), these mice were injected with anti-Ly6G antibodies on P2 and evaluated at the completion of lung development (P30). At this time point, neutrophil-depleted IKTA mice showed normal alveolar structure (Supplemental Figure 8), further supporting the conclusion that neutrophils mediated aberrant lung development in this model.

Next, we investigated whether neutrophils were responsible for downregulation of $E \ln$ and Fbln 5 expression in saccular-stage lungs from IKTA mice (Dox P3-P5). As shown in Figure 6A, anti-Ly6G antibody treatment mitigated downregulation of these elastic fiber assembly components in P5 IKTA lungs. In situ hybridization (RNAscope) confirmed the reduction in Fbln 5 and Eln in P5 IKTA lungs and restoration of expression after anti-Ly6G antibody treatment
(Figure 6B). Consistent with these findings, neutrophil depletion corrected the fragmented and disorganized appearance of elastic fibers around saccular airspaces of P5 IKTA mice (Figure 6C).

Given that elastic fiber assembly components are primarily produced by mesenchymal cells in the developing lung, we tested whether IKTA neutrophils could directly alter mRNA expression of elastic fiber assembly components by these cells. For these experiments, we used FACS to isolate $\mathrm{CD} 45^{+} / \mathrm{CD} 11 \mathrm{~b}^{+} / \mathrm{Ly} 6 \mathrm{G}^{+}$neutrophils from IKTA lungs (Dox P3-P5) and then cocultured these cells with wild-type primary saccular-stage mouse (P5) lung fibroblasts. IKTA neutrophils downregulated $F b \ln 5$ and $E \ln$ expression by saccular lung fibroblasts (Figure 6, D and E), suggesting that recruited neutrophils (or their soluble mediators) were responsible for the altered elastic fiber assembly observed in inflamed saccular-stage lungs.

Neutrophil-derived cytokines do not inhibit elastin assembly. Having identified neutrophils as the primary immune cell type responsible for disrupting elastic fiber assembly in saccular-stage IKTA lungs, we investigated molecular mechanisms by which neutrophils could mediate these effects. Although expression of a variety of inflammatory cytokines was increased in IKTA lungs (Dox P3-P5), only IL-1 $\beta$ expression was altered by neutrophil depletion (Supplemental Figure 9A). Since IL-1 $\beta$ signaling has been shown to alter saccular-stage lung development (18), we tested whether exogenous 

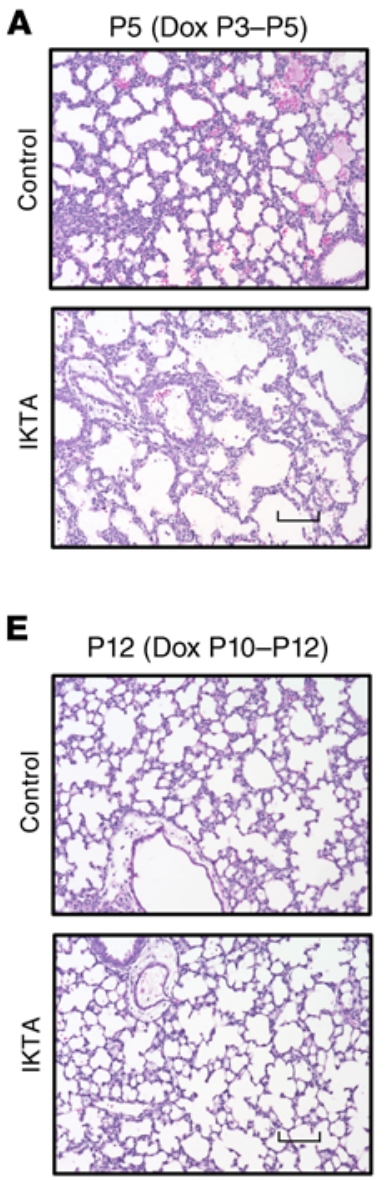

B

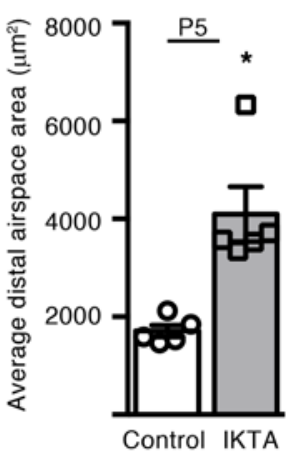

$\mathbf{F}$

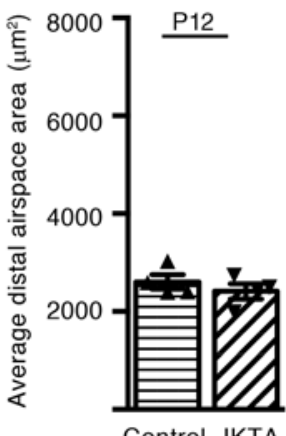

C

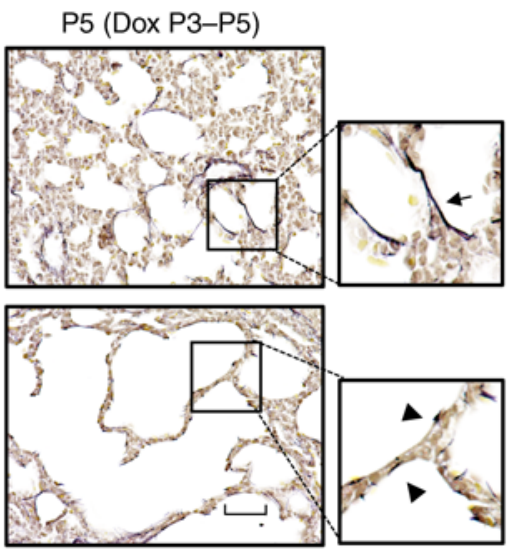

G

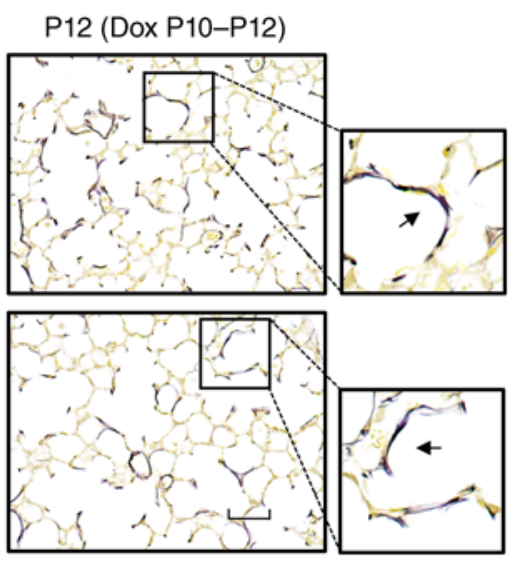

D

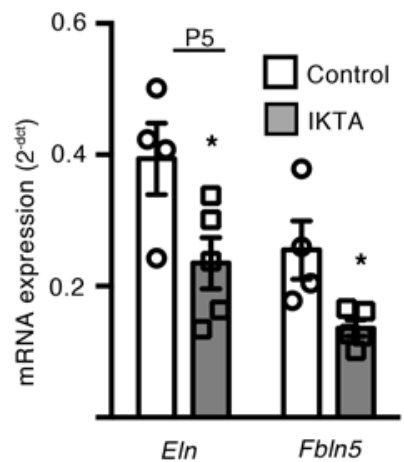

H

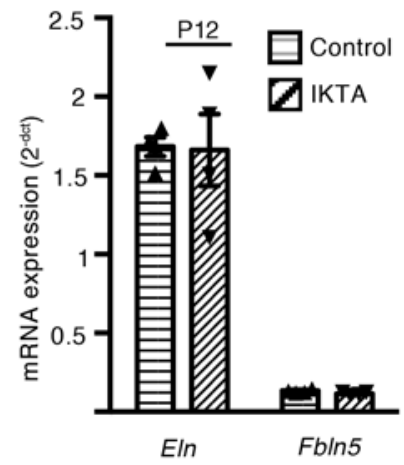

Figure 4. Neutrophilic inflammation disrupts saccular-stage (but not alveolar-stage) elastic fiber assembly and results in a BPD-like phenotype. Dams were treated with Dox from P3 to P5 (saccular stage) or P10 to P12 (alveolar stage), after which lungs from IKTA and littermate control mice were harvested at P5 or P12. (A, B, E, and F) Representative H\&E-stained lung sections ( $\mathbf{A}$ and $\mathbf{E}$ ) and morphometric measurements (B and $\mathbf{F})$ demonstrated dilated terminal saccules in P5 IKTA lungs, whereas P12 IKTA lungs appeared similar to controls. Data are expressed as mean \pm SEM, $n=4-5$ lungs per group, 5-6 imaged lung fields per lung, ${ }^{*} P<0.05$ by 2-tailed Student's $t$ test. ( $\mathbf{C}$ and $\left.\mathbf{G}\right)$ Representative photomicrographs of Hart's elastin-stained sections of P5 (C) and P12 (C) control and IKTA lungs demonstrating disorganized and fragmented elastic fibers in P5 IKTA lungs. Arrows denote normal elastic fibers in controls. Arrowhead denotes fragmented elastic fibers in IKTA lungs. ( $\mathbf{D}$ and $\mathbf{H}$ ) mRNA expression of elastic fiber assembly components Eln and Fbln5 expression in P5 (D) and P12 (H) IKTA lungs. Data are expressed as mean \pm SEM, $n=4-5$ per group, ${ }^{*} P<0.05$ by 2-tailed Student's $t$ test. Scale bar: $100 \mu \mathrm{m}$ in $\mathbf{A}$ and $\mathbf{E}, 50 \mu \mathrm{m}$ in $\mathbf{C}$ and $\mathbf{G}$.

IL-1 $\beta$ could affect elastin and fibulin- 5 expression in cultured saccular-stage mouse lung fibroblasts (Supplemental Figure 9B). Because these data suggested that IL-1 signaling could be responsible for the deleterious effects of neutrophils in saccular-stage IKTA lungs, we generated IKTA mice deficient in IL-1R1, the primary IL-1 signaling receptor; however, IL-1R1 deletion did not rescue the saccular lung phenotype in IKTA mice (Dox P3-P5) (Supplemental Figure 9, C-E) and did not prevent the downregulation of Eln and Fbln5 in IKTA lungs (Supplemental Figure 9F).

Neutrophil elastase downregulates $m R N A$ expression of elastic fiber assembly components by saccular-stage lung fibroblasts. Since neutrophil elastase (NE) was increased in IKTA lungs after Dox treatment (Figure 7A and Supplemental Figure 10A), we wondered whether NE mediates the BPD-like phenotype in P5 IKTA mice. Therefore, we cocultured lung neutrophils $\left(\mathrm{CD} 45^{+} / \mathrm{CD} 1 \mathrm{~b}^{+} / \mathrm{Ly} 6 \mathrm{G}^{+}\right.$cells) obtained by flow sorting from IKTA lungs with wild-type saccular-stage mouse lung fibroblasts in the presence or absence of an irreversible $\mathrm{NE}$ inhibitor (N-methoxysuccinyl-Ala-Ala-Pro-Val-chloromethyl ketone). Treatment with the NE inhibitor normalized expression of $E \ln$ and $F b \ln 5$ by fibroblasts cocultured with IKTA neutrophils (Figure 7B). Further, addition of purified human neutrophil elastase (HNE) to culture media downregulated Eln and Fbln 5 expression by saccular-stage lung fibroblasts (Figure 7C). In comparison, HNE treatment did not affect expression of Eln and Fbln in cultured P12 (alveolar stage) lung fibroblasts (Supplemental Figure 10B), suggesting developmental stage-dependent regulation of mRNA expression for these elastin assembly genes.

$\mathrm{NE}$ is increased in airways of patients with $\operatorname{BPD}(19,20)$, and recent data suggest that exosomes harvested from tracheal aspirates of patients with BPD express active NE on their surface (21). Therefore, we tested whether BPD exosomes could downregulate elastic fiber assembly components by saccular-stage lung fibroblasts. For these experiments, we collected tracheal aspirate exosomes from preterm patients with BPD (or term-born controls) (Supplemental Table 1) and treated saccular-stage mouse lung fibroblasts with pooled exosomes from BPD or control patients. BPD exosomes had higher NE activity (BPD: $0.9 \mu \mathrm{g}$ of HNE-equivalent activity $/ 10^{11}$ exosomes; control: $0.06 \mu \mathrm{g}$ of HNE-equivalent activity $/ 10^{11}$ exosomes) 
A
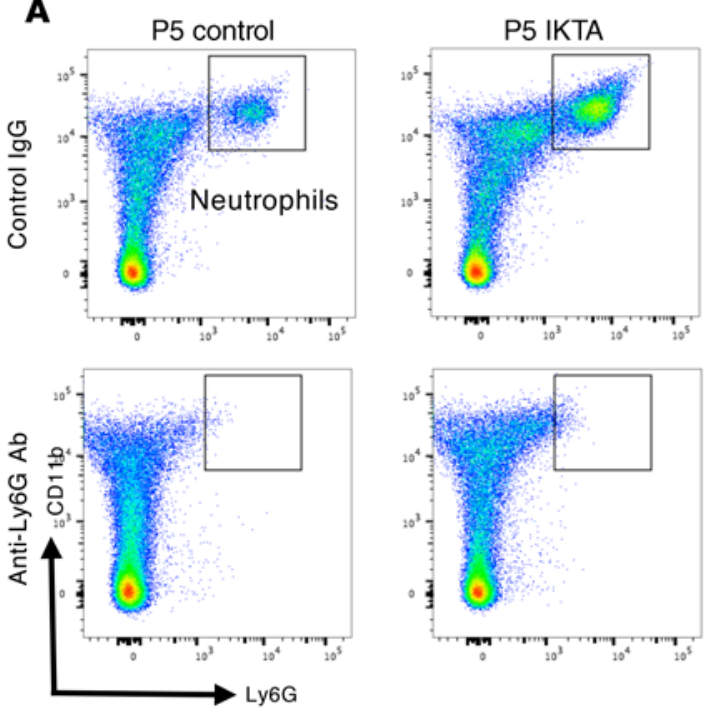

C
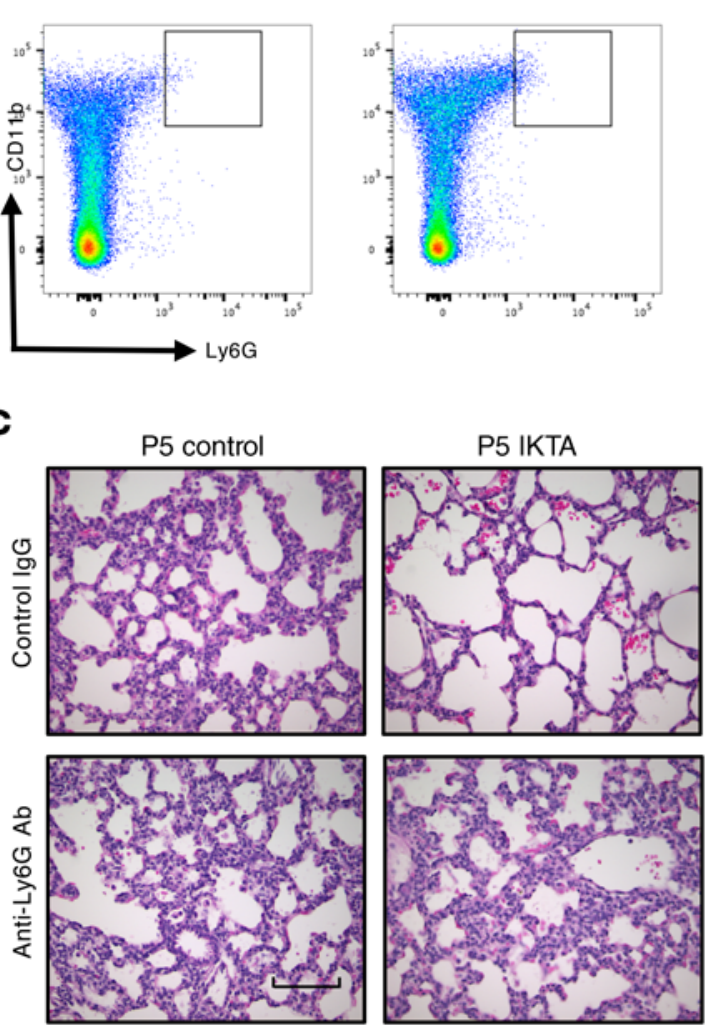

B

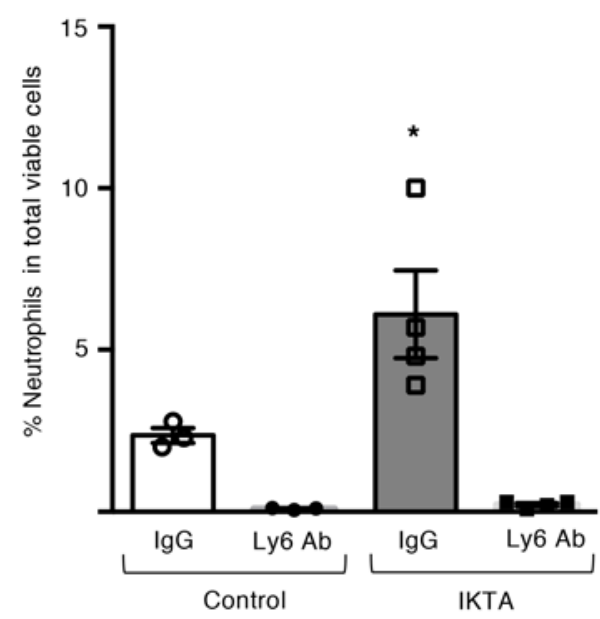

D

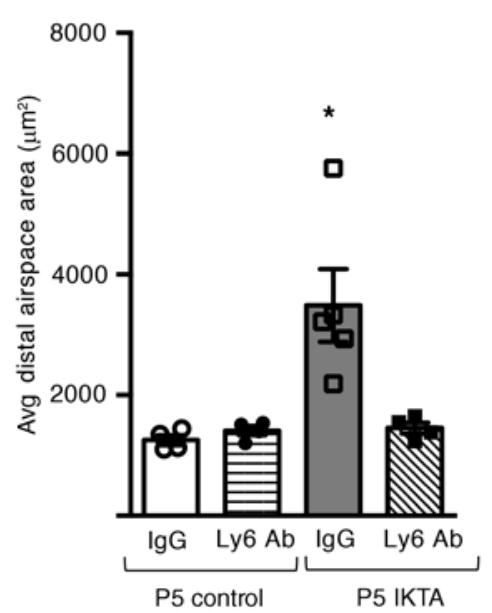

E

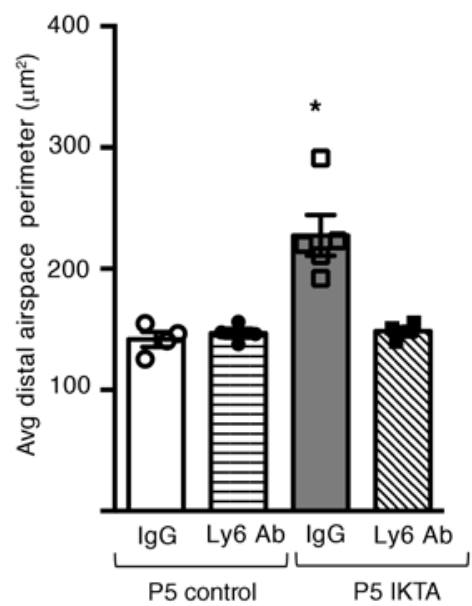

Figure 5. Neutrophil depletion rescues lung development in saccular-stage lungs with NF-кB activation. Control and IKTA pups were administered anti-ly6G antibodies or control IgG by i.p. injection on P2 prior to placing dams on Dox from P3 to P5 for transgene activation. (A and B) Representative flow plots (A) and

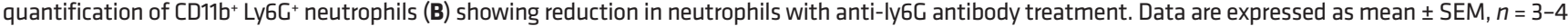
lungs per group, ${ }^{*} P<0.05$ by 1 -way ANOVA and post hoc Tukey test. (C-E) Representative H\&E-stained lung sections (C), morphometric evaluation of average distal airspace area (D), and average distal airspace perimeter (E) in P5 lungs. Data are expressed as mean \pm SEM, $n=4$ lungs per group, 4-6 imaged fields per lung (D and $\mathbf{E}$ ). ${ }^{*} P<0.05$ by 1 -way ANOVA and post hoc Tukey test. Scale bar: $100 \mu \mathrm{m}$ in $\mathbf{C}$.

and downregulated fibroblast Eln and Fbln5 (Figure 7, D and E), thus implicating NE-containing exosomes in blocking production of critical elastic fiber assembly components.

Based on our finding that NE suppresses expression of elastin assembly components in addition to degrading elastin, we tested whether saccular-stage lungs are uniquely susceptible to NE exposure compared with lungs during later stages of development (alveolarization) or after completion of lung development (adults). For these studies, we generated precision-cut lung slices from P5, P12, and adult (2 months old) wild-type mice, and cultured each lung slice with or without $\mathrm{HNE}(4 \mu \mathrm{g} / \mathrm{mL})$ for 4 days. HNE treatment resulted in airspace dilation of P5 lung slices with marked reduction of intact elastic fibers (Supplemental Figure 11). In contrast, at this concentration and exposure time, HNE treatment of P12 or adult lung slices did not alter alveolar architecture and elastin fibers remained intact, thereby supporting the conclusion that saccular-stage lungs are uniquely susceptible to the damaging effects of NE.

$N E$ activates the EGFR/MEK/ERK pathway and inhibits TGF- $\beta$ signaling in saccular-stage lung fibroblasts. To investigate the mechanisms by which NE regulates transcription of critical elastic fiber assembly components in mesenchymal cells, we first tested several candidate pathways that have previously been shown to be activated by $\mathrm{NE}$ and/or downregulate elastin assembly components, including Par-1, Par-2, NF-kB, and p38 MAPK (22-25). Using specific inhibitors of each of these pathways in conjunction with $\mathrm{NE}$ treatment of P5 fibroblasts, we found no impact on NE-mediated suppression of Eln or Fbln 5 expression (Supplemental Figure 12).

Elastases can cleave membrane-bound pro-forms of EGFR ligands, including TGF- $\alpha$, EGF, and heparin-binding EGF (HB-EGF), and release active ligands that transactivate EGFR (26-29). Therefore, 
A

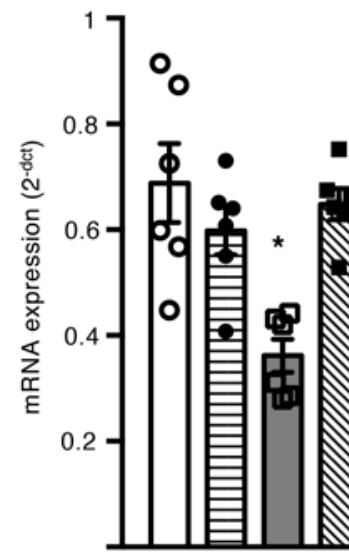

Eln
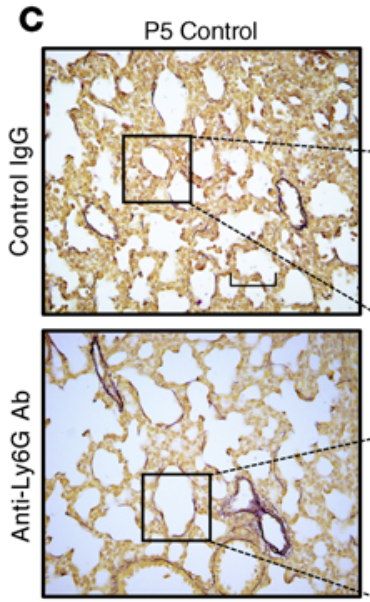

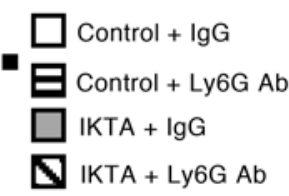
$\circ$

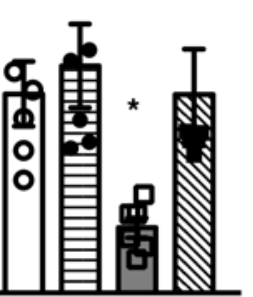

Fbln5
B

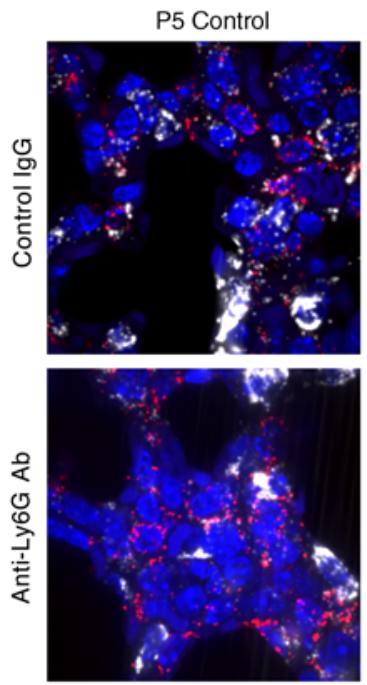

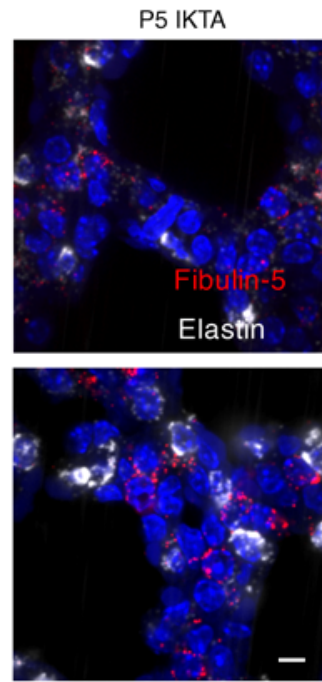

D

E
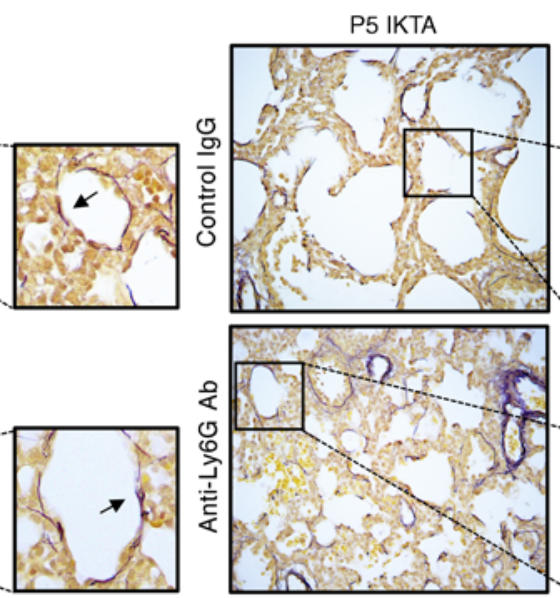

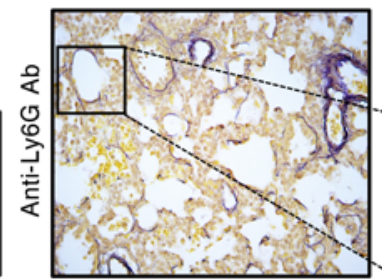

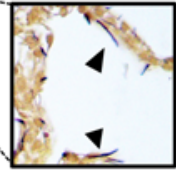

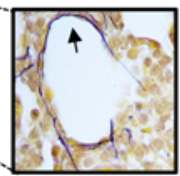

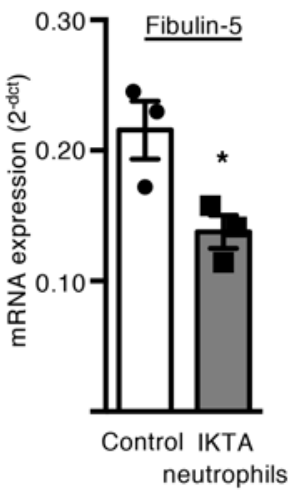

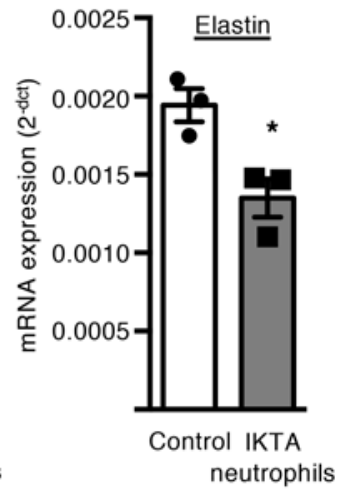

Figure 6. Neutrophil depletion prevents disruption of elastic fibers in saccular-stage IKTA lungs. Control and IKTA pups were administered anti-ly6C antibodies or control IgG IP on P2 prior to placing dams on Dox from P3 to P5 for transgene activation. (A) Expression of Eln and Fbln5 in P5 lungs. Data are expressed as mean \pm SEM, $n=6$ per group, ${ }^{*} P<0.05$ by 1-way ANOVA and post hoc Tukey test. (B) Representative photomicrographs of RNAscope-labeled sections from P5 lungs. Fbln5- and Eln-expressing cells were reduced in IKTA lungs with control lgG treatment, but Fbln5 and Eln expression appeared similar to controls in IKTA mice with anti-Ly6C antibody treatment. (C) Representative Hart's elastin-stained sections from P5 lungs showing normal cord-like structures around airspaces in IKTA mice treated with anti-Ly6C antibodies. Arrows denote elastic fibers around airspaces. Arrowhead denotes fragmented elastic fibers. ( $\mathbf{D}$ and $\mathbf{E})$ CD11 b $^{+}$Ly6C $\mathrm{C}^{+}$neutrophils were isolated from IKTA lungs and cocultured with wild-type saccular-stage lung fibroblast for 4 hours. Expression of $F b l n 5$ (D) and Eln (E) was reduced in saccular-stage lung fibroblasts cocultured with IKTA neutrophils. Data are expressed as mean \pm SEM, $n$ = 3 per group. Data are representative of 3 independent experiments. ( $D$ and $\mathbf{E}),{ }^{*} P<0.05$ by 2-tailed Student's $t$ test. Scale bar: $10 \mu \mathrm{m}$ in $\mathbf{B}, 50 \mu \mathrm{m}$ in $\mathbf{C}$.

we measured EGFR ligands in culture media from P5 fibroblasts after HNE treatment and found that EGF and Hb-EGF were undetectable, but TGF- $\alpha$ was increased by 15 minutes, suggesting proteolytic release (Figure 8A). We then treated saccular-stage lung fibroblasts with recombinant TGF- $\alpha$ and found that expression of Fbln 5 and Eln was reduced (Figure 8, B and C), thereby indicating that HNE-mediated release of TGF- $\alpha$ could downregulate Fbln 5 and Eln expression. To further investigate whether EGFR signaling mediates the effects of HNE on Fbln 5 and Eln expression, we treated P5 fibroblasts with HNE and the selective EGFR tyrosine kinase inhibitor AG1478. As shown in Figure 8D, EGFR inhibition prevented Fbln5 (but not Eln) downregulation. Since MEK/ERK signaling can be induced by EGFR activation, we measured ERK phosphorylation after HNE treatment and found increased ERK phosphorylation by 15 minutes after treatment (Figure 8, E and F). We then treated P5 fibroblasts with the MEK/ERK inhibitor U0126 along with HNE and found that the MEK/ERK inhibitor prevented Fbln 5 downregulation but did not affect Eln expression, similar to EGFR inhibition (Figure 8G). Together, these data showed that NE treatment activated EGFR/MEK/ERK signaling to inhibit Fbln 5 expression in saccular-stage fibroblasts; however, additional pathways are involved in Eln regulation.

To further investigate the mechanisms by which NE suppresses Eln expression in saccular-stage lung fibroblasts, we examined the TGF- $\beta$ pathway, which has been reported to regulate Eln expression in 
A

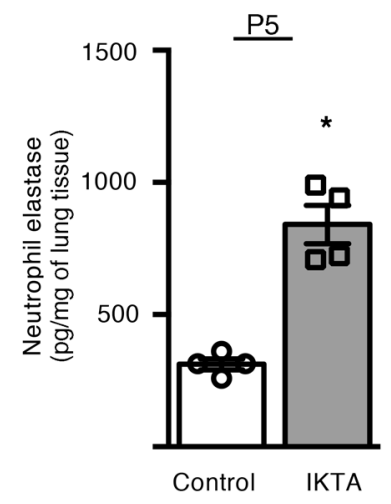

C

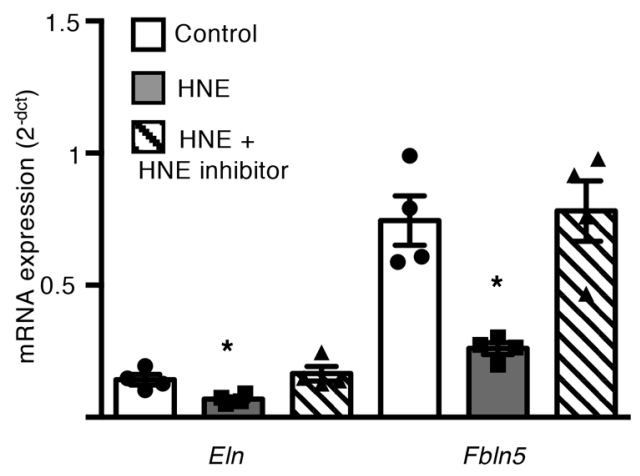

B

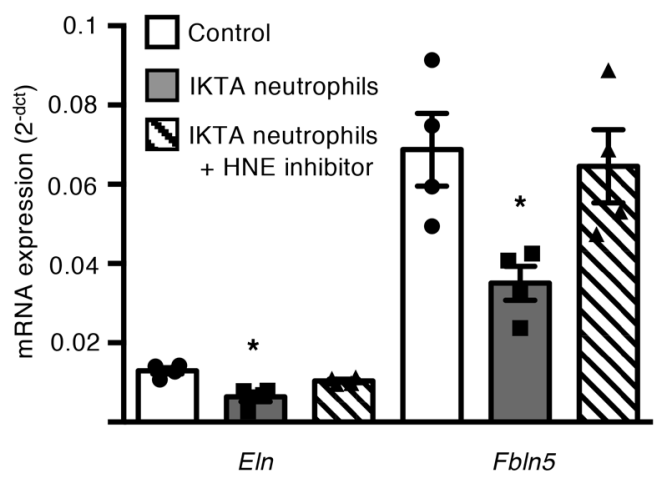

D

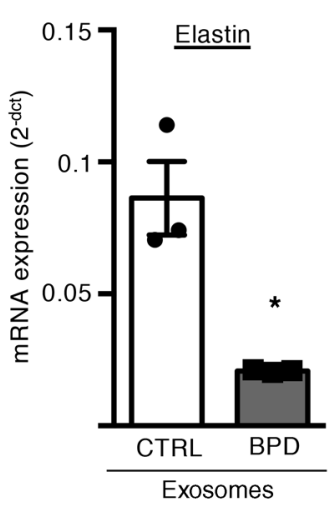

E

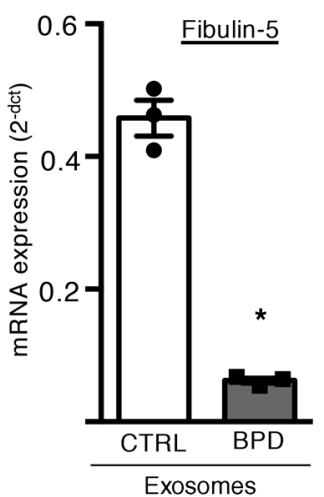

Figure 7. Neutrophil elastase inhibits expression of elastic fiber assembly components by saccular-stage mouse lung fibroblasts. (A) Quantification of neutrophil elastase (NE) by ELISA in P5 control and IKTA lung homogenates. Data are expressed as mean \pm SEM, $n=4-6$ per group, ${ }^{*} P<0.05$ by 2 -tailed Student's $t$ test. (B and C) P5 mouse lung fibroblasts were cultured with or without IKTA neutrophils for 4 hours (B) or purified human neutrophil elastase (HNE) $1 \mu \mathrm{g} / \mathrm{mL}$ for 24 hours (C) and Eln and Fbln5 expression was quantified. Where indicated, cells were also pretreated with $10 \mu \mathrm{M} N$-methoxysuccinyl-Ala-Ala-Pro-Val-chloromethyl ketone (HNE inhibitor). Data expressed as mean \pm SEM, $n=4$ per group. Data are representative of 4 independent experiments. ${ }^{*} P<0.05$ compared with control by 1-way ANOVA and post hoc Tukey test. ( $\mathbf{D}$ and $\mathbf{E})$ Saccular-stage mouse lung fibroblasts were cultured with tracheal aspirate exosomes (10 ${ }^{9}$ exosomes $/ \mathrm{mL}$ ) isolated from preterm infants with severe BPD or from term-born control infants (CTRL) and Eln (D) and Fb/n5 (E) expression was quantified. Data are expressed as mean \pm SEM, $n=3$ per group, ${ }^{*} P<0.05$ by 2 -tailed Student's $t$ test.

human and neonatal rat lung fibroblasts $(28,30)$. Consistent with prior reports, we found that TGF- $\beta$-neutralizing antibodies and the TGF- $\beta$ receptor kinase inhibitor SB 431542 reduced Eln expression, and exogenous TGF- $\beta 1$ treatment upregulated Eln expression in P5 lung fibroblasts (Supplemental Figure 13, A and B). To test whether NE-dependent suppression of Eln expression could be mediated by inhibition of canonical TGF- $\beta$ signaling, P5 lung fibroblasts were transfected with a Smad2/3-LUC reporter plasmid prior to HNE treatment. HNE treatment reduced reporter activity in control and TGF- $\beta 1$-stimulated cells (Figure 9, A and B), along with downregulation of TGF- $\beta$-regulated genes, including Ctgf, Serpine-1, and Tbsp1 (Figure 9C). We then pretreated P5 lung fibroblasts with TGF- $\beta 1$ prior to HNE treatment and found that addition of TGF- $\beta 1$ prevented HNE-induced downregulation of Eln expression and modestly increased $F b \ln 5$ expression (Figure 9, D and E). Collectively, these data suggest that interruption of canonical TGF- $\beta$ signaling in saccular lung fibroblasts by HNE is important for downregulation of Eln expression.

\section{Discussion}

Our studies identified a window of susceptibility in the saccular stage of lung development (E18-P5 in mice, 23-34 weeks gestation in humans), during which neutrophilic inflammation can disrupt formation of the elastic fiber network in the distal lung, leading to lifelong impairment in lung structure and function. The saccular stage marks a critical period during development in which the preterm lung undergoes numerous changes required for successful transition to postnatal life, including production and assembly of nascent elastic fibers in the extracellular matrix surrounding developing saccules. Neutrophilic inflammation, which could occur as a result of infection (chorioamnionitis) or injury to the developing lung, prevents normal assembly of elastic fibers around terminal airspaces and leads to BPD-like alveolar simplification, followed by a sustained reduction in lung function, with airspace enlargement (emphysema) and loss of alveolar attachments surrounding small airways that progresses with advancing age. This phenotype is at least partially driven by $\mathrm{NE}$, which has a dual role of downregulating expression of key elastin assembly components, particularly Fbln5 and $E l n$, as well as matrix destruction.

Given that lung function trajectories appear to be established early in childhood $(2,31)$, injuries that reduce lung function during early life could limit the peak lung function attainable in adulthood, thereby representing a major risk factor for COPD 
A

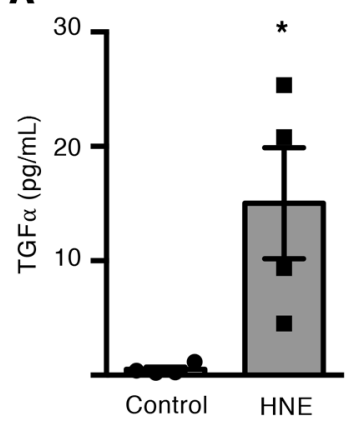

D
B

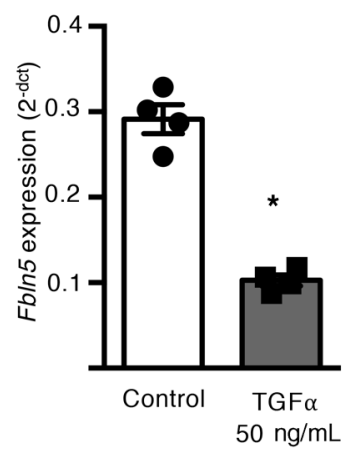

C

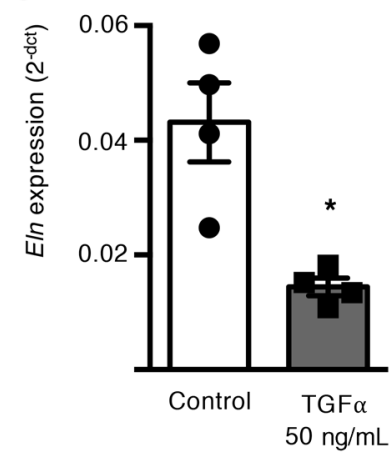

E
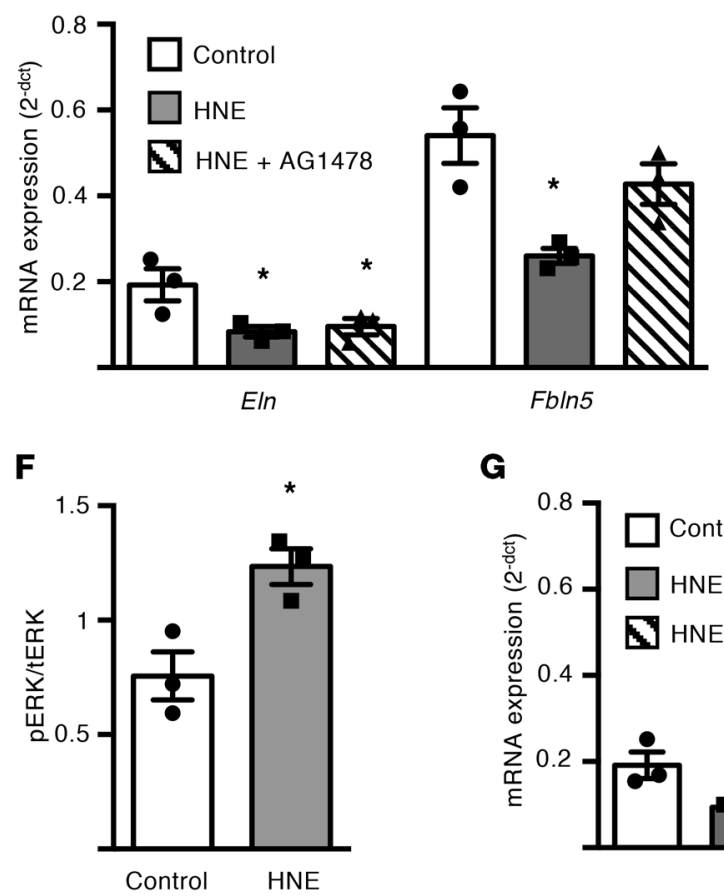

G

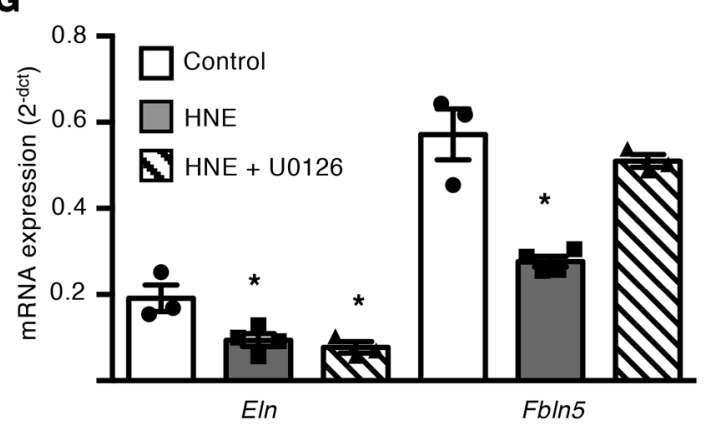

Figure 8. Neutrophil elastase downregulates mRNA expression of fibulin-5 in saccular-stage mouse lung fibroblasts through EGFR/MEK/ERK signaling. P5 mouse lung fibroblasts were grown to confluence and cultured with or without purified human neutrophil elastase (HNE) at 1 or $4 \mu \mathrm{g} / \mathrm{mL}$ or TCF- $\alpha(50 \mathrm{ng} / \mathrm{mL})$ for up to 24 hours. In some experiments, fibroblasts were treated with AG1478 $20 \mu \mathrm{M}$ (EGFR inhibitor) or U0126 $25 \mu \mathrm{M}$ (MEK inhibitor) prior to HNE treatment. (A) TCF- $\alpha$ ELISA from conditioned media samples after 15 minutes of HNE treatment of saccular stage lung fibroblasts. Data are expressed as mean \pm SEM, $n$ $=4$ per group. $(\mathbf{B}$ and $\mathbf{C})$ Quantification of $F b \ln 5$ (B) and $E \ln (\mathbf{C})$ expression by qPCR in saccular-stage fibroblasts 24 hours after treatment with TCF- $\alpha$. Data are expressed as mean \pm SEM, $n=4$ per group. (D) Expression of Eln and Fbln5 in fibroblasts treated with HNE \pm AG1478 $20 \mu M$ for 24 hours. Data are expressed as mean \pm SEM, $n=3-4$ per group. (E and $\mathbf{F}$ ) Western blot analysis of phospho-Erk1/2 and total-Erk1/2 in cell lysates from fibroblasts treated with HNE for 15 minutes. Data are expressed as mean \pm SEM, $n=3$ per group. (C) Expression of Eln and Fbln5 in fibroblasts treated with HNE \pm U0126 $25 \mu M$ for 24 hours. Data are expressed as mean \pm SEM, $n=3-4$ per group. Data are representative of 3 independent experiments. ${ }^{*} P<0.05$ compared with control by 2 -tailed student's $t$ test (A, B, C, and $\mathbf{F}) ;{ }^{*} P<0.05$ compared with control by 1-way ANOVA and post hoc Tukey test (D and $\left.\mathbf{G}\right)$.

$(2,32)$. This idea is supported by multiple studies showing that extremely preterm human infants who develop BPD continue to have lung function abnormalities during their growing years (4-6, $33,34)$. In addition to prematurity, maternal smoking, childhood lower respiratory infections, malnutrition, and air pollution may also contribute to the low-function lung phenotype in adulthood $(35,36)$. Therefore, although the saccular stage of lung development appears to be uniquely susceptible to neutrophilic inflammation, other insults during alveolarization and lung growth may also alter lung-function trajectory. An important question for future studies is whether subtle inflammatory insults during the saccular stage, which are insufficient to cause BPD, can instigate enough disruption in elastin fiber assembly to restrict peak lung function and predispose to COPD.

Neutrophils have been implicated as an important cell type in COPD pathogenesis for many years. The "protease/antiprotease hypothesis" for COPD development is based on the phenotype seen in smokers with alpha-1 antiprotease deficiency, in which continued lung inflammation results in an imbalance of matrix-degrading enzymes (particularly NE), favoring destruction of gas exchanging units and accelerated decline in lung function $(37,38)$. It was unexpected, however, that neutrophils 
A

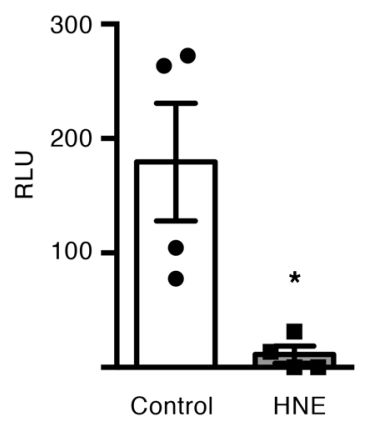

B

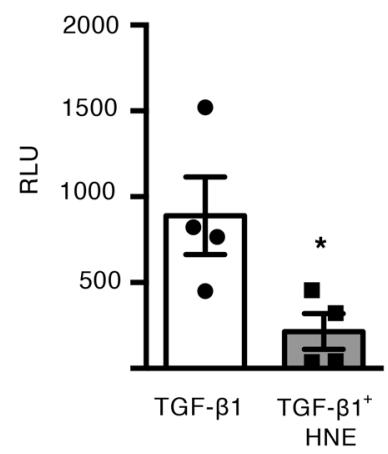

C

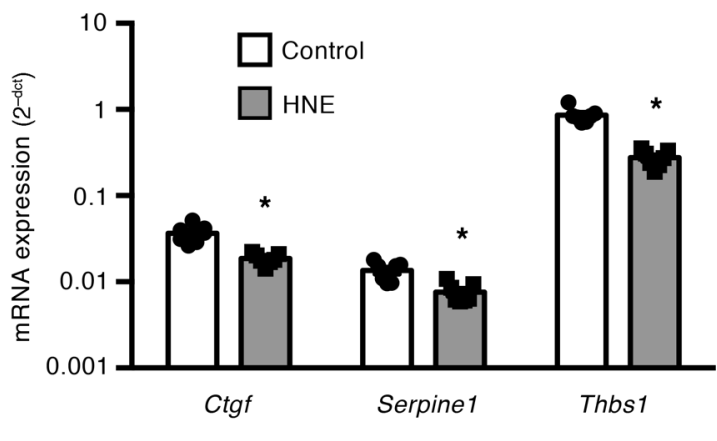

D

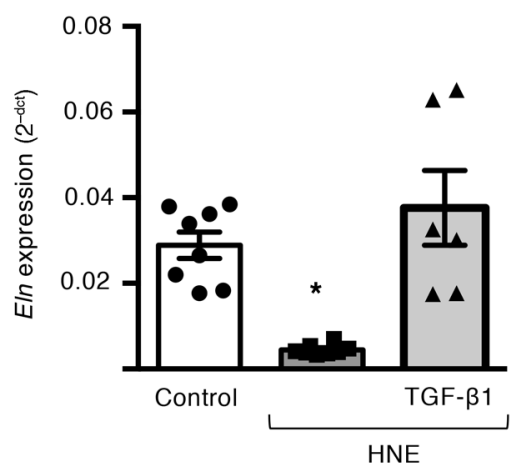

E

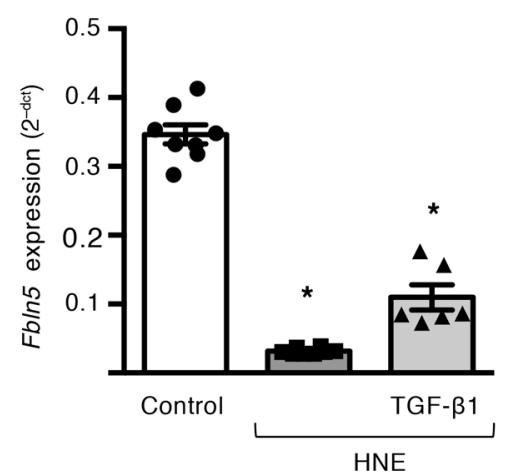

Figure 9. Neutrophil elastase downregulates TGF- $\beta$ signaling and reduces elastin expression in saccular-stage lung fibroblasts. (A and B) P5 mouse lung fibroblasts were transfected with a Smad LUC reporter (SBE4-LUC) and treated with or without HNE ( $\mu \mathrm{g} / \mathrm{mL})$ and/or recombinant TCF- $\beta 1$ ( $0.1 \mathrm{ng} /$ $\mathrm{mL}$ ) for 12 hours. Luminescence measurements are reported as relative light units (RLU) above background (untransfected control) in (A) control cells and (B) TCF- $\beta 1$-treated cells. Data are expressed as mean $\pm \mathrm{SEM}, n=4$ per group. ${ }^{*} P<0.05$ by 2-tailed Student's $t$ test. (C-E) Mouse lung fibroblasts were cultured with or without HNE $(1 \mu \mathrm{g} / \mathrm{mL})$ and TGF- $\beta 1(0.1 \mathrm{ng} / \mathrm{mL})$ for 24 hours. (C) mRNA expression of Ctgf, Serpine1, and Thbs 1 in fibroblasts treated with or without HNE. Data are from 3 independent experiments and expressed as mean \pm SEM, $n=7$ per group. ${ }^{*} P<0.05$ by 2 -tailed Student's $t$ test. (D and E) mRNA expression of Eln (D) and Fbln5 (E) in saccular-stage fibroblasts treated with HNE \pm recombinant TGF- $\beta 1$. Data are from 3 independent experiments and expressed as mean \pm SEM, $n=6-8$ per group, ${ }^{*} P<0.05$ compared with control by 1-way ANOVA and post hoc Tukey test.

could also play a critical role in early-life predisposition to COPD through a separate function of inhibiting elastin assembly. Our studies showed that impaired elastin fiber assembly around developing saccules led to emphysematous airspace dilation and a reduction in alveolar attachments surrounding distal airways, both of which affect elastic recoil and could contribute to airflow limitation. Although the effects of emphysema on lung function are well known, the loss of alveolar attachments and their contribution to airflow limitation in COPD are underappreciated (39, 40). Reduction in alveolar attachments is thought to untether small airways from adjacent alveolar tissue, thereby contributing to dynamic airflow limitation through reduced radial tension and premature closure of small airways during expiration $(14,41)$.

Neutrophils are critical mediators of innate immune signaling in the lung and can contribute to inflammatory lung injury in the neonatal and adult lung (42-44). For example, neutrophils mediate alveolar simplification in neonatal rats exposed to hyperoxia (45). In adult IKTA mice, treatment with Dox drives robust transgene activation and a neutrophil-predominant inflammatory cell influx, resulting in acute lung injury with substantial mortality beginning around day 7 of transgene induction (13). Longer-term treatment with low-dose Dox causes a COPD-like phenotype in IKTA mice with emphysema, elastin degradation, and small airway wall remodeling by 3-4 months of treatment (46). In our studies, neutrophil depletion rescued the saccular-stage lung phenotype in IKTA mice, thus confirming neutrophils as the culprit for mediating the effects of inflammation on elastic fiber assembly. While it is tautology that NE can cleave elastin and other components of the lung extracellular matrix, more recent studies indicate that NE can also modulate gene expression $(26,47,48)$. Depending on the cell type and context of signaling, NE may either upregulate $(47,48)$ or downregulate gene expression $(28,49)$. In saccular- but not alveolar-stage mouse lung fibroblasts, we found that NE suppressed expression of Eln and Fbln5, thus suggesting the possibility that distinct subpopulations of fibroblasts (with differential susceptibility to NE) produce elastin and elastin assembly components in the saccular and alveolar stage. Although this issue requires further investigation, our findings indicate that mesenchymal cells in the saccular-stage lung are particularly susceptible to NE.

Since exosomes harvested from tracheal aspirates of infants with BPD contain active NE on their surface and administration of these exosomes can induce a BPD-like phenotype in mice (21), we tested whether BPD exosomes could affect expression of elastin assembly components by saccular-stage fibroblasts. In keeping with a dual function of NE in saccular-stage lung development, we found that BPD patient exosomes inhibited $E \ln$ and $F b \ln 5$ expression by 
saccular-stage lung fibroblasts. Collectively, these findings build on prior work demonstrating susceptibility of elastin organization in the saccular-stage lung to inflammation (12), and highlight what we believe to be a novel role for NE in dysregulating elastic fiber assembly in the saccular lung mesenchyme.

In our studies, we showed that downregulation of Fbln 5 by NE in saccular-stage lung fibroblasts was mediated through the EGFR/ MEK/ERK signaling pathway. EGFR signaling is important for early lung morphogenesis (50), and reduced EGFR signaling increases apoptosis and prevents lung growth in newborn mice exposed to mechanical ventilation with hyperoxia (51). However, aberrant EGFR activation can also be detrimental to the neonatal lung (52) and EGFR ligands, including EGF and TGF- $\alpha$, are increased in lungs of infants with $\operatorname{BPD}(52,53)$. In this regard, transgenic overexpression of human TGF- $\alpha$ in the early postnatal mouse lung alters elastic fiber organization and lung structures, leading to COPDlike changes in the adult lung, similar to those seen in IKTA mice with transgene activation during the saccular stage (54). Although previous studies have shown that NE can cleave cell membranebound pro-forms of EGFR ligands, including EGF, HB-EGF, and TGF- $\alpha$ (26-29), we found that only TGF- $\alpha$ (but not EGF or HB-EGF) was increased in conditioned media from NE-treated P5 lung fibroblasts, suggesting a likely role for soluble TGF- $\alpha$ in activating EGFR in NE-treated saccular-stage lung fibroblasts. However, given the complexity of the in vivo setting, other EGFR ligands or nonligand-mediated activation of EGFR could also play a role in Fbln 5 downregulation in lungs of IKTA mice.

In contrast to $F b \ln 5$, Eln expression by saccular-stage lung fibroblasts appears to be primarily regulated by pathways other than EGFR, particularly the TGF- $\beta$ pathway $(30,55)$. Prior reports indicate that autocrine TGF- $\beta$ signaling can increase fibroblast Eln expression by stabilizing Eln mRNA (56). We found that HNE downregulated autocrine TGF- $\beta$-dependent Smad signaling in P5 fibroblasts, and addition of exogenous TGF- $\beta$ restored $E$ ln expression after HNE treatment. Although full elucidation of mechanisms connecting NE and intracellular signaling pathways in saccular-stage lung fibroblasts requires additional study, NE appears to alter a variety of signaling pathways in fibroblasts, including EGFR/MEK/ERK and TGF- $\beta$, which can perturb elastin assembly and alter lung development during the saccular stage.

Although our studies specifically focused on the role of $\mathrm{NE}$, we acknowledge that other neutrophil products may also affect the abnormal saccular-stage lung phenotype that we observed in IKTA mice. IL-1 $\beta$ is increased in the lungs of preterm infants at risk for chronic lung disease $(57,58)$, and transgenic expression of human IL-1 $\beta$ in the mouse lung alters saccular-stage lung development (18). In the hyperoxia model of BPD, abrogating IL-1 $\beta$-mediated IL-1R1 signaling prevents abnormalities in saccular-stage lung development (59). Although neutrophils are an important source of IL-1 $1 \beta$, genetic deficiency of IL-1R1 in our in vivo studies did not prevent abnormalities in elastin assembly or rescue the abnormal saccular-stage lung phenotype that we observed in lungs of IKTA mice. Thus, specific molecular mechanisms contributing to impaired lung development may depend on the context and type of inflammatory stimulus.

In summary, our findings delineate the saccular stage as a critical period for laying down the elastic fiber scaffold in the lung. NE secreted by activated neutrophils downregulated production of critical elastic fiber assembly components and altered assembly of elastic fibers in the saccular stage, thereby resulting in persistent deficits in lung function and a COPD-like adult lung phenotype. These findings may have relevance in understanding the developmental origins of COPD and other adult lung diseases.

\section{Methods}

Mouse models. All mice used for experiments in this study were on a C57BL/6 background. Transgenic mice that express a FLAG-tagged form of activated human IKK- $\beta$ containing S177E and S188E mutations under the control of a tetracycline operator minimal CMV promoter and a tetracycline-controlled transcription silencer under the control of the Clara cell $10 \mathrm{kDa}$ (CC10) promoter have been previously described (FLAG-IKK- $\beta$ mice) (13). To activate NF- $\kappa B$ in the airway epithelium, hemizygous FLAG-IKK- $\beta$ male mice were crossed with homozygous female mice expressing reverse tetracycline transactivator (rtTA) under control of the Club cell-specific protein promoter (CCSP-rtTA mice). Approximately half of the pups from these matings expressed all 3 transgenes and activated NF- $\mathrm{BB}$ in the airway epithelium after treatment with Dox. These mice were designated IKTA mice, and the remaining pups in each litter that expressed only the CCSP-rtTA transgene were used as littermate controls. For transgene activation in neonatal lungs, lactating dams were administered $100 \mathrm{mg} / \mathrm{L}$ of Dox (Sigma-Aldrich) in drinking water from P3 to P5 (saccular-stage transgene activation) or from P10 to P12 (alveolar-stage transgene activation). In some experiments, IKTA mice deficient in IL-1R1 were also used. IL-1R1 ${ }^{-/-}$mice (B6.129S7-Ilr1 ${ }^{\text {tmIImx }} / \mathrm{J}$, stock 003245) were purchased from The Jackson Laboratory. FLAG-IKK- $\beta$ and CC10-rtTA mice were separately backcrossed to IL-1R1 $1^{-/-}$mice to generate FLAG-IKK- $\beta$ and CCSP-rtTA mice deficient in IL-1R1 respectively. IL-1R1 $1^{-/}$FLAG-IKK- $\beta$ and IL1R1 ${ }^{--}$CCSP-rtTA mice were then crossed to generate IKTA mice (and littermate controls) deficient in IL-1R1.

To block neutrophil influx, neonatal IKTA mice (and littermate controls) were i.p. administered a neutralizing anti-Ly6G antibody (20 $\mu \mathrm{g}$ of antibody per pup; Bio X Cell, clone 1A8, BP0075-1) or an IgG1a isotype control antibody (20 $\mu$ g of antibody per pup; Bio X Cell, BP0089) on P2 prior to Dox administration from P3 to P5. Lungs were then harvested on P5 for additional studies.

Lung function measurements. After transgene activation from P3 to P5 or from P10 to P12, IKTA and littermate control mice were serially followed, and lung function measurements were quantified at various time points using the FlexiVent apparatus (SCIREQ) and established methods $(60,61)$. Briefly, mice were anesthetized with pentobarbital sodium $(85$ $\mathrm{mg} / \mathrm{kg}$ ), and an 18-gauge tracheostomy tube was placed in the trachea. Mice were then mechanically ventilated using the SCIREQ FlexiVent apparatus with 150 breaths/min and a tidal volume of $10 \mathrm{~mL} / \mathrm{kg}$ body weight prior to lung function measurements. Respiratory system elastance and compliance were captured using the Flexivent "Snapshot model," and tissue elastance (H) was captured using a constant phase model to obtain a parametric distinction between airway and tissue mechanics.

Precision-cut lung slices. Precision-cut lung slices were made from neonatal mouse lungs at P5 or P12 or from adult mouse lungs using previously published methods $(62,63)$. Briefly, after euthanasia, lungs were perfused with cold PBS, after which a blunt-tipped catheter was inserted into the trachea. Low melting temperature agarose ( $2 \%$ agarose in DMEM/F121:1) was then instilled into the lung via the tracheal catheter, after which the lung was removed en bloc and placed on ice. Using a Leica 
VT1000E vibrating microtome tissue slicer, $250 \mu \mathrm{m}$ thick sections were cut from the left lobe. Tissue slices were cultured in DMEM/F12 with or without $\mathrm{HNE}$ treatment for up to 96 hours at $37^{\circ} \mathrm{C}$, after which lung slices were harvested and fixed in $10 \%$ formalin for additional studies.

Flow cytometry. Lung tissue was digested using collagenase XI (0.7 $\mathrm{mg} / \mathrm{mL}$; Sigma-Aldrich, C7657), type IV DNase $(30 \mu \mathrm{g} / \mathrm{mL}$; SigmaAldrich, D5025), and Dispase $(0.25 \mathrm{mg} / \mathrm{mL})$ and filtered through a $70 \mu \mathrm{m}$ filter to obtain a single-cell suspension. Cells were labeled with conjugated primary antibodies and analyzed using a 3-laser BD Fortessa analytical flow cytometer (BD Biosciences) and FlowJo software (BD Biosciences). Conjugated primary antibodies used for flow cytometry were as follows: CD45-BV510 (BioLegend, clone 30-F11), CD11b-PE-Cy7 (BioLegend, clone M1/70), Ly6G-APC-Cy7 (BioLegend, clone 1A8), CD64-APC (BioLegend, clone X54-5/7.1), F4/80-PE-Cy5 (BioLegend, clone BM8), Siglec F-PE (BD Pharmingen, clone E50-2440), Ly6C-PerCP-Cy5.5 (BD Pharmingen, clone AL-21), and I-A/I-E MHC-II-FITC (BD Pharmingen, clone 2G9). DAPI (Sigma-Aldrich) labeling was used to assess cell viability.

To isolate saccular lung neutrophils, P5 lung tissue was first digested and processed to a single-cell suspension as above. Cells were then labeled with conjugated primary antibodies and subjected to FACS on a FACSAria 11 cell sorter (BD Biosciences) with FACSDiVa v6.1 software (BD Biosciences) to isolate $\mathrm{CD} 45^{+} \mathrm{CD} 11 \mathrm{~b}^{+} \mathrm{Ly}-6 \mathrm{G}^{+}$neutrophils that were collected for in vitro experiments.

Histology and immunostaining. Whole mouse lungs and neonatal mouse precision-cut lung slices were fixed overnight with $10 \%$ formalin prior to paraffin-embedding and staining sections with H\&E using standard methods. Images of $\mathrm{H} \& \mathrm{E}$ sections were then imaged using a brightfield microscope. For immunoperoxidase staining, after rehydration and antigen retrieval using citrated buffer at $\mathrm{pH} 6.5,5 \mu \mathrm{m}$ paraffin sections were sequentially incubated with primary and biotinylated secondary antibodies. Immunostaining was then detected using ABC kits and NovaRED reagents (Vector Laboratories). Slides were counterstained with hematoxylin prior to imaging using a brightfield microscope. For immunofluorescent staining, lung sections were first permeabilized with $0.1 \%$ Triton X-100 (Thermo Fisher Scientific). Then, after overnight incubation with primary antibodies, sections were incubated with Alexa-conjugated secondary antibodies as indicated and imaged using a fluorescent microscope. Elastic fibers were localized in lung sections using Hart's elastin stain using previously described methods (12).

Morphometry and image analysis. For morphometric evaluations, brightfield images of H\&E-stained lung sections were captured as TIFF files and imported into Image (NIH) for analysis. Morphometric measurements including MLI, average distal airspace area, and average distal airspace perimeter were quantified using previously described methods $(12,64)$. For alveolar attachment counts, alveolar attachments were identified and counted for each small airway and normalized to airway basement membrane length. For Gr-1-positive cell counts, $\times 40$ images of lung sections immunostained with an anti-Gr-1 antibody (R\&D Systems, MAB1037) were captured and cell counts per high-power field directly quantified. SMA immunostaining was done on lung sections using an anti- $\alpha$-SMA antibody (Abcam, ab5694).

For pulmonary vessel density, lung sections were immunostained with anti-vWF antibody (Dako, A0082) and vWF-stained blood vessels less than $50 \mu \mathrm{m}$ in diameter were quantified per high-power field.

RNA in situ hybridization and immunofluorescence imaging. RNAscope (ACD) was used to perform RNA in situ hybridization according to the manufacturer's instructions as previously described $(63,65)$. RNAscope probes to the following mouse genes were used for experiments: Fbln 5 (ACD, 493621) and $E \ln$ (ACD, 319361). Immunofluorescent images were then captured using an automated TiE inverted fluorescence microscope and $\mathrm{a} \times 100$ Plain Apo objective (Nikon Instruments, Inc.).

To identify proliferating parenchymal cells, lung sections were immunostained with anti-PCNA (Cell Signaling Technology, 2586) and anti-pro-SPC (Abcam, ab90716) antibodies and imaged using a Keyence BZ-X710 fluorescent microscope with BZ-X viewer software (Keyence) with a $\times 40$ objective. Immunofluorescent images were then imported into ImageJ for quantification of all PCNA-positive cells (all proliferating cells) and pro-SPC/PCNA dual-positive cells (proliferating type 2 alveolar epithelial cells) in the lung. Apoptotic cells were detected in FFPE lung sections using the in situ cell-death detection kit (MilliporeSigma) per the manufacturer's suggested methods.

Mouse lung fibroblast isolation and culture. Fibroblasts were isolated from P5 and P12 mouse lungs using previously described methods (12). Briefly, after perfusion with sterile PBS, mouse lungs were harvested, dissected, and enzymatically digested in DMEM containing collagenase type I $(0.5 \mathrm{mg} / \mathrm{mL}$; Sigma-Aldrich) and collagenase type IA (0.5 $\mathrm{mg} / \mathrm{mL}$; Sigma-Aldrich), followed by trituration to mechanically dissociate lung tissue. The resulting cell suspension was sequentially filtered through 100, 70, and $20 \mu \mathrm{m}$ sterile filters. The filtrate from the $20 \mu \mathrm{m}$ filter was plated in $100 \mathrm{~mm}$ cell-culture dishes and cultured in DMEM supplemented with $10 \%$ FCS. For all experiments, passage 3 or 4 fibroblasts were first plated in 12- or 24-well plates or chamber slides and cultured for 72 hours. After overnight serum starvation, cells were exposed to various treatment conditions for 4-48 hours in serum-free conditions prior to harvesting for RNA extraction or protein quantification.

Smad reporter assay. Saccular-stage mouse lung fibroblasts were grown to $40 \%-50 \%$ confluence in 24 -well cell culture plates. Cells were transfected with $3 \mu \mathrm{g}$ of SBE4-LUC reporter, an LUC reporter vector containing 4 copies of the Smad binding site (Addgene) (66), and $0.3 \mu \mathrm{g}$ of the Renilla LUC control vector with Superfect transfection reagent (QIAGEN) using the manufacturer's suggested methods. Where appropriate, 24 hours after transfection, cells were treated with TGF- $\beta 1(0.1 \mathrm{ng} / \mathrm{mL})$ and/or HNE $(1 \mu \mathrm{g} / \mathrm{mL})$ for 12 hours. Luminescence was then measured using the dual-LUC assay reagent on a GloMax plate reader (Promega).

Real-time quantitative PCR. Total RNA was extracted from neonatal lungs and lung fibroblasts using TRIzol reagent (Life Science Technologies) using standard methods (67). First-strand cDNA was synthesized using oligo-dT primers and Moloney murine leukemia virus reverse transcriptase (Superscript II; Invitrogen). PCR primers were designed using PrimerQuest design tool (Integrated DNA Technologies). Two-step PCR was performed using an Applied Biosystems StepOne-Plus Real Time PCR system (Applied Biosystems) and SYBR green detection system (Bio$\mathrm{Rad})$. Gene expression was normalized to $\beta$-actin in each sample. Fold change in mRNA expression was calculated using the $2^{\text {-ddCT }}$ method (68).

Western blot and densitometry. Mouse lung tissue and cultured lung fibroblasts were homogenized in RIPA buffer with a protease and phosphatase inhibitor cocktail (Sigma-Aldrich). Total protein was quantified using a bicinchoninic acid protein assay kit (Thermo Fisher Scientific). Equal amounts of protein from each sample were loaded on a NuPAGE polyacrylamide gel (Invitrogen) and transferred to a nitrocellulose membrane (Invitrogen). After blocking with Odyssey blocking buffer (LI-COR), membranes were sequentially incubated with primary and Odyssey IRDye 800CW and 680RD secondary antibodies. Primary antibodies used for Western blot analysis were anti-Phospho-Erk1/2 anti- 
body (Cell Signaling Technology, 9101), anti-Total-Erk1/2 (Cell Signaling Technology, 9107) antibody, and anti- $\beta$-actin antibody (Sigma-Aldrich, A5316). Blots were detected using the Odyssey CLx imaging system (LI-COR). For densitometry analysis, TIFF images of blots were imported into Image J software and quantified using standard methods.

ELISA. Mouse NE ELISA was done with kits purchased from R\&D systems (MELA20). TGF- $\alpha$ ELISA was done using kits from MyBioSource (MBS2508394). All assays were done using the manufacturer's recommended methods. For all assays, samples were measured in duplicate. OD values were imported into GraphPad Prism software, and concentrations determined and normalized to total protein content where indicated.

Human tracheal aspirate exosome isolation and quantification of elastase activity. Tracheal aspirates were collected from 11 patients with BPD and 10 term-born control infants as previously described (21). Aspirate fluid was subjected to centrifugation at $10,000 \mathrm{~g}$ for 1 hour to pellet cells and smaller particles. Subsequently, the supernatant fluid was subjected to ultracentrifugation (150,000 $g$ for 2 hours) for exosome extraction. Exosomes collected from individual patients within each group (BPD and control) were pooled, quantified, and resuspended in PBS prior to use in experiments. Elastase content of exosome samples were measured in duplicate using the EnzChek elastase assay kit (Molecular Probes) per the manufacturer's suggested methods. Known concentrations of HNE were run in parallel to generate a standard curve. Exosome elastase activity was expressed in HNE equivalents.

Statistics. All statistical analysis was done using GraphPad Prism version 6.0. Comparisons between groups were performed using a 2-tailed Student's $t$ test or a 1-way ANOVA followed by the Tukey post hoc test. A $P$ value less than 0.05 was used to determine statistical significance.

Study approval. The experiments in this study were conducted in accordance with the guidelines outlined by the US Public Health Service Policy on the Humane Care and Use of Laboratory Animals. All animal experimental protocols were approved by the IACUC at
Vanderbilt University Medical Center (protocol M1700066). Collection of neonatal tracheal aspirates was performed with written informed consent and approved by the IRB at the University of Alabama at Birmingham (IRB-140926006).

\section{Author contributions}

JTB, EJP, JMS, RVM, SD, SG, DSN, PMG, CSJ, WH, MX, PCD, AC, SK, DCN, BWR, and JAK designed and performed experiments and analyzed data. CVL, KD, AG, and JEB provided the BPD and control patient exosomes. JTB, TSB, LRY, and SHG conceived of and designed the experiments. JTB and TSB drafted and revised the manuscript. All authors reviewed and approved the final manuscript.

\section{Acknowledgments}

This work is supported by NIH grants K12 HD087023 (Research Scholar, JTB), K08 HL133484 (JTB), K08 HL127102 (EJP), K12 HD087023 (Research Scholar, JMS), K08 HL143051 (JMS), K08 HL141652 (CVL), R01 HL119503 (LRY), K24HL143281 (LRY), R35 HL135710 (JEB), R01 GM108807 (SHG), P01 HL092870 (TSB); the Francis Family Foundation (JMS); American Heart Association grant AHA 17SDG32720009 (CVL); and US Department of Veteran Affairs grants IK2BX003841 (BWR) and I01 BX002378 (TSB). Experiments were performed in part through the use of the Vanderbilt Cell Imaging Shared Resource (supported by NIH grants CA68485, DK20593, DK58404, DK59637, and EY08126) and the VUMC Flow Cytometry Shared Resource (supported by the Vanderbilt Ingram Cancer Center, P30 CA68485 and the Vanderbilt Digestive Disease Research Center, DK058404).

Address correspondence to: John T. Benjamin, Monroe Carell Jr. Children's Hospital, Vanderbilt University Medical Center, 2215 B Garland Avenue, 1125C Light Hall, Nashville, Tennessee 37232, USA. Phone: 615.343.6495; Email: john.benjamin@vumc.org.
1. Lange $P$, et al. Lung-function trajectories leading to chronic obstructive pulmonary disease. $\mathrm{NEnglJ}$ Med. 2015;373(2):111-122.

2. Martinez FD. Early-life origins of chronic obstructive pulmonary disease. $\mathrm{NEnglJMed}$. 2016;375(9):871-878.

3. Postma DS, Bush A, van den Berge M. Risk factors and early origins of chronic obstructive pulmonary disease. Lancet. 2015;385(9971):899-909.

4. Islam JY, Keller RL, Aschner JL, Hartert TV, Moore PE. Understanding the short- and long-term respiratory outcomes of prematurity and bronchopulmonary dysplasia. Am J Respir Crit Care Med. 2015;192(2):134-156.

5. Doyle LW, et al. Increasing airway obstruction from 8 to 18 years in extremely preterm/low-birthweight survivors born in the surfactant era. Thorax. 2017;72(8):712-719.

6. Baraldi E, Carraro S, Filippone M. Bronchopulmonary dysplasia: definitions and long-term respiratory outcome. Early Hum Dev. 2009;85(suppl 10):S1-S3.

7. Kotecha SJ, et al. Effect of preterm birth on later FEV1: a systematic review and meta-analysis. Tho$\operatorname{rax} .2013 ; 68(8): 760-766$.

8. Wong PM, et al. Emphysema in young adult survivors of moderate-to-severe bronchopulmonary dysplasia. Eur Respir J. 2008;32(2):321-328.
9. Kallapur SG, Jobe AH. Contribution of inflammation to lung injury and development. Arch Dis Child Fetal Neonatal Ed.2006;91(2):F132-F135.

10. Watterberg KL, Demers LM, Scott SM, Murphy S. Chorioamnionitis and early lung inflammation in infants in whom bronchopulmonary dysplasia develops. Pediatrics. 1996;97(2):210-215.

11. Van Marter LJ, et al. Chorioamnionitis, mechanical ventilation, and postnatal sepsis as modulators of chronic lung disease in preterm infants. JPediatr. 2002;140(2):171-176.

12. Benjamin JT, et al. Epithelial-derived inflammation disrupts elastin assembly and alters saccular stage lung development. Am J Pathol. 2016;186(7):1786-1800.

13. Cheng DS, et al. Airway epithelium controls lung inflammation and injury through the NF-kappa B pathway. JImmunol. 2007;178(10):6504-6513.

14. Baraldo S, Turato G, Saetta M. Pathophysiology of the small airways in chronic obstructive pulmonary disease. Respiration. 2012;84(2):89-97.

15. Shapiro SD, Endicott SK, Province MA, Pierce JA, Campbell EJ. Marked longevity of human lung parenchymal elastic fibers deduced from prevalence of D-aspartate and nuclear weapons-related radiocarbon. J Clin Invest. 1991;87(5):1828-1834.

16. Mariani TJ, Sandefur S, Pierce RA. Elastin in lung development. Exp Lung Res. 1997;23(2):131-145.

17. Abman SH. Bronchopulmonary dysplasia: "a vascular hypothesis”. Am J Respir Crit Care Med. 2001;164(10 Pt 1):1755-1756.

18. Bry K, Whitsett JA, Lappalainen U. IL-1beta disrupts postnatal lung morphogenesis in the mouse. Am J Respir Cell Mol Biol. 2007;36(1):32-42.

19. Speer CP. Inflammation and bronchopulmonary dysplasia: a continuing story. Semin Fetal Neonatal Med. 2006;11(5):354-362.

20. Merritt TA, et al. Elastase and alpha 1-proteinase inhibitor activity in tracheal aspirates during respiratory distress syndrome. Role of inflammation in the pathogenesis of bronchopulmonary dysplasia. JClin Invest. 1983;72(2):656-666.

21. Genschmer KR, et al. Activated PMN exosomes: pathogenic entities causing matrix destruction and disease in the lung. Cell. 2019;176(1-2):113-126.e15.

22. Mihara K, Ramachandran R, Renaux B, Saifeddine M, Hollenberg MD. Neutrophil elastase and proteinase-3 trigger G protein-biased signaling through proteinase-activated receptor-1 (PAR1). J Biol Chem. 2013;288(46):32979-32990.

23. Zhao P, et al. Neutrophil elastase activates protease-activated receptor-2 (PAR2) and transient receptor potential vanilloid 4 (TRPV4) to cause inflammation and pain. J Biol Chem. 
2015;290(22):13875-13887.

24. Kuang PP, et al. NF-kappaB induced by IL-1beta inhibits elastin transcription and myofibroblast phenotype. Am J Physiol Cell Physiol. 2002;283(1):C58-C65.

25. Chen HC, et al. Neutrophil elastase induces IL-8 synthesis by lung epithelial cells via the mitogen-activated protein kinase pathway. J Biomed Sci. 2004;11(1):49-58.

26. Kohri K, Ueki IF, Nadel JA. Neutrophil elastase induces mucin production by ligand-dependent epidermal growth factor receptor activation. Am J Physiol Lung Cell Mol Physiol. 2002;283(3):L531-L540.

27. DiCamillo SJ, et al. Elastase-released epidermal growth factor recruits epidermal growth factor receptor and extracellular signal-regulated kinases to downregulate tropoelastin mRNA in lung fibroblasts. J Biol Chem. 2002;277(21):18938-18946.

28. DiCamillo SJ, et al. Neutrophil elastase-initiated EGFR/MEK/ERK signaling counteracts stabilizing effect of autocrine TGF-beta on tropoelastin mRNA in lung fibroblasts. Am J Physiol Lung Cell Mol Physiol. 2006;291(2):L232-L243.

29. Liu J, Rich CB, Buczek-Thomas JA, Nugent MA, Panchenko MP, Foster JA. Heparin-binding EGFlike growth factor regulates elastin and FGF-2 expression in pulmonary fibroblasts. Am JPhysiol Lung Cell Mol Physiol. 2003;285(5):L1106-L1115.

30. Kuang PP, Zhang XH, Rich CB, Foster JA, Subramanian M, Goldstein RH. Activation of elastin transcription by transforming growth factor-beta in human lung fibroblasts. Am JPhysiol Lung Cell Mol Physiol. 2007;292(4):L944-L952.

31. Stocks J, Sonnappa S. Early life influences on the development of chronic obstructive pulmonary disease. Ther Adv Respir Dis. 2013;7(3):161-173.

32. McGrath-Morrow SA, Collaco JM. Bronchopulmonary dysplasia: what are its links to COPD? Ther Adv Respir Dis. 2019;13:1753466619892492.

33. Vollsæter M, Røksund OD, Eide GE, Markestad T, Halvorsen T. Lung function after preterm birth: development from mid-childhood to adulthood. Thorax. 2013;68(8):767-776.

34. Bhandari A, McGrath-Morrow S. Long-term pulmonary outcomes of patients with bronchopulmonary dysplasia. Semin Perinatol. 2013;37(2):132-137.

35. Svanes C, et al. Early life origins of chronic obstructive pulmonary disease. Thorax. 2010;65(1):14-20.

36. Bush A. Lung development and aging. Ann Am Thorac Soc. 2016;13(suppl 5):S438-S446.

37. Shapiro SD, Goldstein NM, Houghton AM, Kobayashi DK, Kelley D, Belaaouaj A. Neutrophil elastase contributes to cigarette smoke-induced emphysema in mice. Am J Pathol. 2003;163(6):2329-2335.

38. Cavarra E, Martorana PA, Gambelli F, de Santi M, van Even P, Lungarella G. Neutrophil recruit- ment into the lungs is associated with increased lung elastase burden, decreased lung elastin, and emphysema in alpha 1 proteinase inhibitordeficient mice. Lab Invest. 1996;75(2):273-280.

39. Nagai A, Yamawaki I, Takizawa T, Thurlbeck WM. Alveolar attachments in emphysema of human lungs. Am Rev Respir Dis. 1991;144(4):888-891.

40. Saetta M, et al. Loss of alveolar attachments in smokers. A morphometric correlate of lung function impairment. Am Rev Respir Dis. 1985;132(4):894-900.

41. Kim V, Rogers TJ, Criner GJ. New concepts in the pathobiology of chronic obstructive pulmonary disease. Proc Am Thorac Soc. 2008;5(4):478-485.

42. Grommes J, Soehnlein O. Contribution of neutrophils to acute lung injury. MolMed.2011;17(3-4):293-307.

43. Aulakh GK. Neutrophils in the lung: "the first responders”. Cell Tissue Res. 2018;371(3):577-588.

44. Pierce MR, Bancalari E. The role of inflammation in the pathogenesis of bronchopulmonary dysplasia. Pediatr Pulmonol.1995;19(6):371-378.

45. Auten RL, Mason SN, Tanaka DT, Welty-Wolf K, Whorton MH. Anti-neutrophil chemokine preserves alveolar development in hyperoxia-exposed newborn rats. Am J Physiol Lung Cell Mol Physiol. 2001;281(2):L336-L344.

46. Zaynagetdinov R, et al. Chronic NF-kB activation links COPD and lung cancer through generation of an immunosuppressive microenvironment in the lungs. Oncotarget. 2016;7(5):5470-5482.

47. Devaney JM, Greene CM, Taggart CC, Carroll TP, O’Neill SJ, McElvaney NG. Neutrophil elastase up-regulates interleukin-8 via toll-like receptor 4. FEBS Lett. 2003;544(1-3):129-132.

48. Geraghty P, et al. Neutrophil elastase up-regulates cathepsin B and matrix metalloprotease-2 expression. JImmunol. 2007;178(9):5871-5878.

49. Domon H, et al. Neutrophil elastase subverts the immune response by cleaving Toll-like receptors and cytokines in pneumococcal pneumonia. Front Immunol. 2018;9:732.

50 . Warburton D, et al. Epigenetic role of epidermal growth factor expression and signalling in embryonic mouse lung morphogenesis. Dev Biol. 1992;149(1):123-133.

51. Alejandre Alcazar MA, et al. Elafin treatment rescues EGFR-Klf4 signaling and lung cell survival in ventilated newborn mice. Am JRespir Cell Mol Biol. 2018;59(5):623-634.

52. Strandjord TP, Clark JG, Guralnick DE, Madtes DK. Immunolocalization of transforming growth factor-alpha, epidermal growth factor (EGF), and EGF-receptor in normal and injured developing human lung. Pediatr Res. 1995;38(6):851-856.

53. Stahlman MT, Orth DN, Gray ME. Immunocytochemical localization of epidermal growth factor in the developing human respiratory system and in acute and chronic lung disease in the neonate. $L a b$ Invest. 1989;60(4):539-547.

54. Le Cras TD, et al. Transient induction of TGF-alpha disrupts lung morphogenesis, causing pulmonary disease in adulthood. Am J Physiol Lung Cell Mol Physiol. 2004;287(4):L718-L729.

55. Sproul EP, Argraves WS. A cytokine axis regulates elastin formation and degradation. Matrix Biol. 2013;32(2):86-94.

56. McGowan SE, McNamer R. Transforming growth factor-beta increases elastin production by neonatal rat lung fibroblasts. Am J Respir Cell Mol Biol. 1990;3(4):369-376.

57. Kotecha S, Wilson L, Wangoo A, Silverman M, Shaw RJ. Increase in interleukin (IL)- 1 beta and IL- 6 in bronchoalveolar lavage fluid obtained from infants with chronic lung disease of prematurity. Pediatr Res. 1996;40(2):250-256.

58. Rindfleisch MS, Hasday JD, Taciak V, Broderick K, Viscardi RM. Potential role of interleukin- 1 in the development of bronchopulmonary dysplasia. JInterferon Cytokine Res. 1996;16(5):365-373.

59. Liao J, et al. The NLRP3 inflammasome is critically involved in the development of bronchopulmonary dysplasia. Nat Commun. 2015;6:8977.

60. Polosukhin VV, et al. Intratracheal bleomycin causes airway remodeling and airflow obstruction in mice. Exp Lung Res. 2012;38(3):135-146.

61. Lawson WE, et al. Endoplasmic reticulum stress enhances fibrotic remodeling in the lungs. Proc Natl Acad Sci U S A. 2011;108(26):10562-10567.

62. Alsafadi HN, et al. An ex vivo model to induce early fibrosis-like changes in human precision-cut lung slices. Am J Physiol Lung Cell Mol Physiol. 2017;312(6):L896-L902.

63. Sucre JMS, et al. Hyperoxia injury in the developing lung Is mediated by mesenchymal expression of Wnt5A. Am J Respir Crit Care Med. 2020;201(10):1249-1262.

64. Plosa EJ, et al. $\beta 1$ Integrin regulates adult lung alveolar epithelial cell inflammation. JCI Insight. 2020;5(2):129259.

65. Wang F, et al. RNAscope: a novel in situ RNA analysis platform for formalin-fixed, paraffin-embedded tissues. J Mol Diagn. 2012;14(1):22-29.

66. Zawel L, et al. Human Smad3 and Smad4 are sequence-specific transcription activators. Mol Cell. 1998;1(4):611-617.

67. Benjamin JT, Smith RJ, Halloran BA, Day TJ, Kelly DR, Prince LS. FGF-10 is decreased in bronchopulmonary dysplasia and suppressed by Toll-like receptor activation. Am JPhysiol Lung Cell Mol Physiol. 2007;292(2):L550-L558.

68. Livak KJ, Schmittgen TD. Analysis of relative gene expression data using real-time quantitative PCR and the 2(-Delta Delta C(T)) Method. Methods. 2001;25(4):402-408. 
